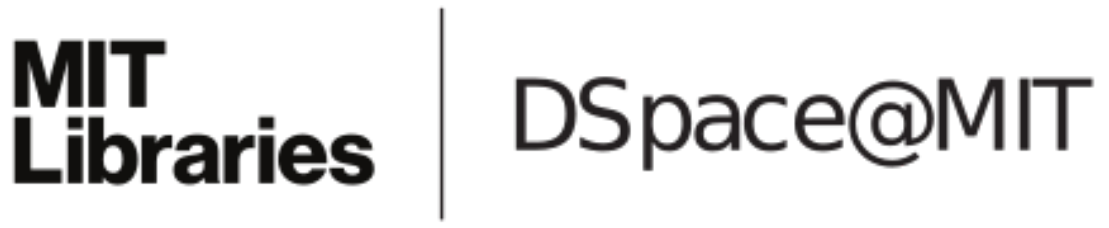

\author{
MIT Open Access Articles
}

Design for additive manufacturing: cellular structures in early-stage aerospace design

The MIT Faculty has made this article openly available. Please share how this access benefits you. Your story matters.

As Published: https://doi.org/10.1007/s00158-019-02305-8

Publisher: Springer Berlin Heidelberg

Persistent URL: https://hdl.handle.net/1721.1/131440

Version: Author's final manuscript: final author's manuscript post peer review, without publisher's formatting or copy editing

Terms of Use: Article is made available in accordance with the publisher's policy and may be subject to US copyright law. Please refer to the publisher's site for terms of use. 


\section{Design for Additive Manufacturing: Cellular Structures in Early- Stage Aerospace Design}

Cite this article as: Max M. J. Opgenoord and Karen E. Willcox, Design for Additive Manufacturing: Cellular Structures in Early-Stage Aerospace Design, Structural and Multidisciplinary Optimization doi: 10.1007/s00158-019-02305-8

This Author Accepted Manuscript is a PDF file of a an unedited peer-reviewed manuscript that has been accepted for publication but has not been copyedited or corrected. The official version of record that is published in the journal is kept up to date and so may therefore differ from this version.

Terms of use and reuse: academic research for non-commercial purposes, see here for full terms. http://www.springer.com/gb/open-access/authors-rights/aam-terms-v1 


\title{
Design for Additive Manufacturing: Cellular Structures in Early-Stage Aerospace Design
}

\author{
Max M. J. Opgenoord • Karen E. Willcox
}

Received: date / Accepted: date

\begin{abstract}
The immense design freedom offered by additive manufacturing yields tremendous benefits, but it also raises the question of how to best go about designing components to exploit this design freedom. In early design phases, design tools need to utilize this freedom, but do so with low computational cost and with a good estimate of the final weight to correctly assess the part's influence on the overall product design. This is especially important for aerospace designs. In this paper, a design methodology is devised for cellular structures to be manufactured using additive manufacturing techniques, specifically for such early design phases. This design methodology uses adaptive meshing techniques to design the topology of the cellular structure, after which the struts of that cellular structure are optimized separately to reduce the dimensionality of the problem. The method is demonstrated for a small generic bracket and an aircraft bracket design from the literature. For these problems, our method is fast enough to evaluate many thousands of design options. This results in identifying promising candidate designs for further detailed design work.
\end{abstract}

Keywords Design for additive manufacturing · cellular structures · aerospace · early-stage design

M. Opgenoord

Department of Aeronautics \& Astronautics

Massachusetts Institute of Technology

Cambridge, MA, USA

E-mail: mopg@mit.edu

K. Willcox

Institute for Computational Engineering and Sciences

University of Texas at Austin

Austin, TX, USA

E-mail: kwillcox@ices.utexas.edu

\section{Introduction}

Additive manufacturing builds up products in a layerwise fashion, eliminating most of the geometric limitations present in subtractive manufacturing methods. However, designing for additive manufacturing is still not a straightforward problem. This is especially true for the design of lightweight structures, such as cellular structures. Typically, only the detailed design of those structures is considered, using expensive design tools. However, in early design phases, we also require a good estimate of the weight and performance of such a part, but typically have lower computational budget available. This paper describes a methodology to design the topology of the cellular structure such that it is aligned with the stress direction and such that its density is based on the stress magnitude throughout the part - all with low computational effort to permit incorporation in early design phases.

Research into design for additive manufacturing has mostly focused on the detailed design of (smaller) structural components, where the allowable design space and the loads are known and fixed. An example of such a detailed design study is the work by Aage et al. (2017) who performed a 1-billion-element topology optimization study on the internal wing structure of a commercial jetliner, where the resulting wing structure mirrored typical bone structures found in a bird wing. However, when additive manufacturing is used for larger parts and where the part's structural properties affect its loading (e.g., a wing structure), the effect of additive manufacturing on the overall product design also has to be considered in early-stage design phases. This is especially important in aerospace designs, for instance when additive manufacturing techniques are used for the wing - for which several design methods 
for additive manufacturing have been published (Stanford and Dunning, 2015; Kolonay and Kobayashi, 2015; Townsend et al., 2018). In early-stage design phases, potentially thousands of designs need to be evaluated quickly on the system level in a multi-disciplinary fashion (Martins, 2017). In such a system level design study, the loads into the structure are continually changing, requiring repeated redesigns of the structural components. Structural design tools for early-stage design phases therefore need to be low-cost, but at the same time result in designs that are sufficiently good representations of the final (detailed) design. Most topology optimization methods are more suited for detailed design studies due to their cost and lower-resolution representations of the same optimization problem can be far off from the final result. We therefore develop a design methodology specifically for early-stage design phases, with low computational cost and a good approximation of the final structural properties.

An example of a bio-inspired structure that is of interest to structural engineers is the cellular structure. ${ }^{1}$ Cellular structures occur in almost any living tissue in nature, displaying optimized properties such as stiffness-to-weight ratio, strength-to-weight ratio (Gibson, 1997; Mazur et al., 2016), thermal conductivity, acoustic absorption, and gas permeability (Rehme, 2010) Furthermore, cellular structures can be more resistant to buckling (Sigmund et al., 2016), and are attractive from a fail-safe design perspective as it is typically straight forward to design for multiple load paths through a part. They can also be tailored to exhibit a specific (even negative) Poisson's ratio (Clausen et al., 2015).

In order to design structural parts for additive manufacturing in detailed design studies, topology optimization methods are often used. Such topology optimization methods are based on structural finite element methods where either each part of the domain is assigned a density determined by an optimizer in so-called Solid-Isotropic-Material-with-Penalization (SIMP) methods (Bendsøe and Sigmund, 2013; Bendsøe and Kikuchi, 1988), or where the optimizer can control the parameters of a level-set to yield a sharp boundary for the final part (Sethian and Wiegmann, 2000; Osher and Santosa, 2001; Van Dijk et al., 2013). To more efficiently generate cellular structures using such approaches, the result from a topology optimization problem on a coarse mesh can be projected down to finer length scales to yield a cellular structure (Pantz and Trabelsi, 2008; Groen

\footnotetext{
1 With "cellular structure" we refer to a structure that consists of nodes and struts connecting those struts, where all struts can have different cross-sectional areas. When there is a repeating pattern to a cellular structure, it is typically referred to as a "lattice structure."
}

and Sigmund, 2017). Alternatively, notions of structural components such as struts can be embedded in a continuum topology optimization problem (Guo et al., 2014; Norato et al., 2015; Watts and Tortorelli, 2018).

A different approach designs cellular structures directly, through for instance ground structure optimization methods (Dorn et al., 1964; Hemp and Chan, 1966; Vanderplaats and Moses, 1972; Kirsch, 1989). A similar approach was used by Zhou (2012) to design truss-like continuum structures, optimizing for the density and orientation of members of a truss-like structure. These methods numerically approximate optimal Michel structures (Michell, 1904) using a finite number of struts, yielding a structure more similar to an animal bone structure. However, these methods are inherently limited by the original mesh on which the ground structure is generated. This can be partially alleviated by tailoring a limited number of unit cells to the stress state in different parts of the structure and using those to generate a new ground structure mesh (Graf et al., 2009).

Although additive manufacturing offers larger design freedom compared to conventional manufacturing techniques, some manufacturing constraints remain. Because the parts are built up in a layer-wise fashion they need support from the layer below them, limiting the overhang angles between subsequent layers. Even for powder-based techniques, such overhang limits usually remain to prevent warping. Selective Laser Sintering (SLS), however, does not have such overhang constraints as it is powder-based and the temperature gradients are lower. Critical overhang angles are typically $45^{\circ} \pm 10^{\circ}$ (Rehme, 2010; Schmidtke et al., 2011), but this number is strongly dependent on the manufacturing process and the material. Furthermore, the geometric resolution is limited - and dependent on manufacturing process and material - which also limits the minimum wall thickness.

Including such manufacturing constraints in structural optimization techniques is critical, otherwise the optimized model needs to be manually altered to adhere to the manufacturing constraints, resulting in a loss of optimality - as illustrated in Fig. 1. Moreover, dedicated design for additive manufacturing - including its manufacturing constraints - is of major importance for the introduction of additive manufacturing as an economically viable production technique (Vaneker, 2017). The manufacturing constraint most commonly used in topology optimization is a minimum or maximum length scale constraint (Poulsen, 2003; Guest et al., 2004; Guest, 2008). Recently, topology optimization methods started considering other manufacturing constraints, such as overhang constraints (Gaynor and Guest, 2016; Langelaar, 2016; Qian, 2017). For cellular structures, the com- 


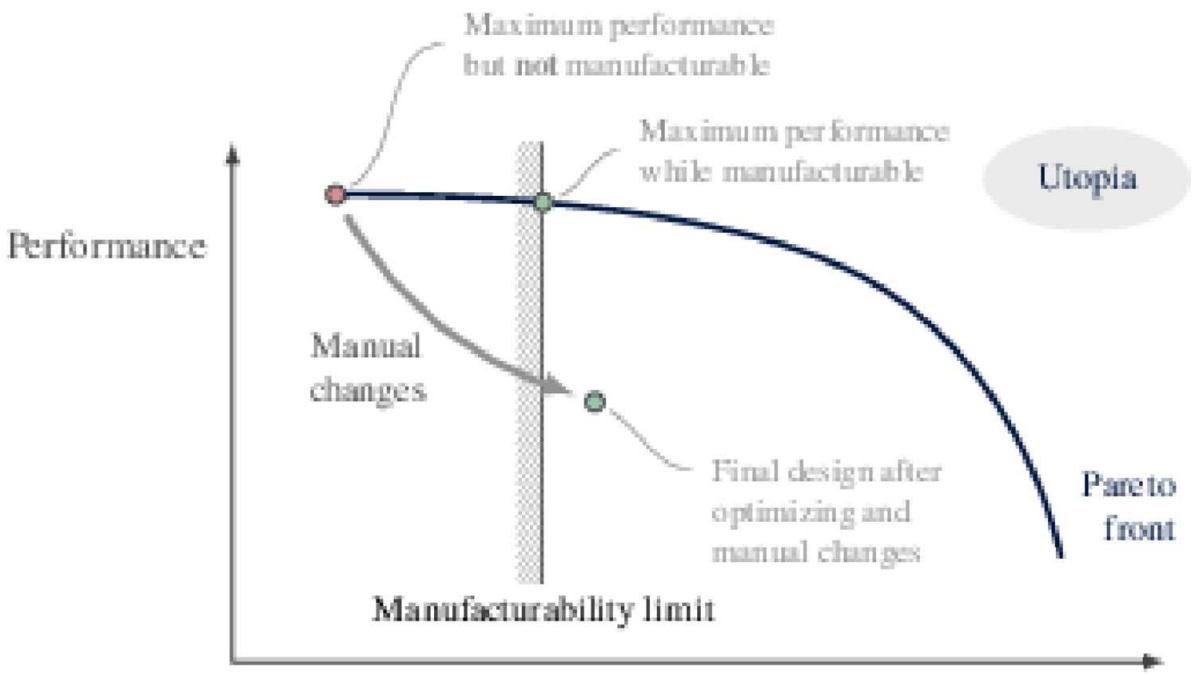

Ease of manufacturing

Fig. 1: Manufacturing constraints need to be included in the optimization to ensure the final manufactured part has optimal performance.

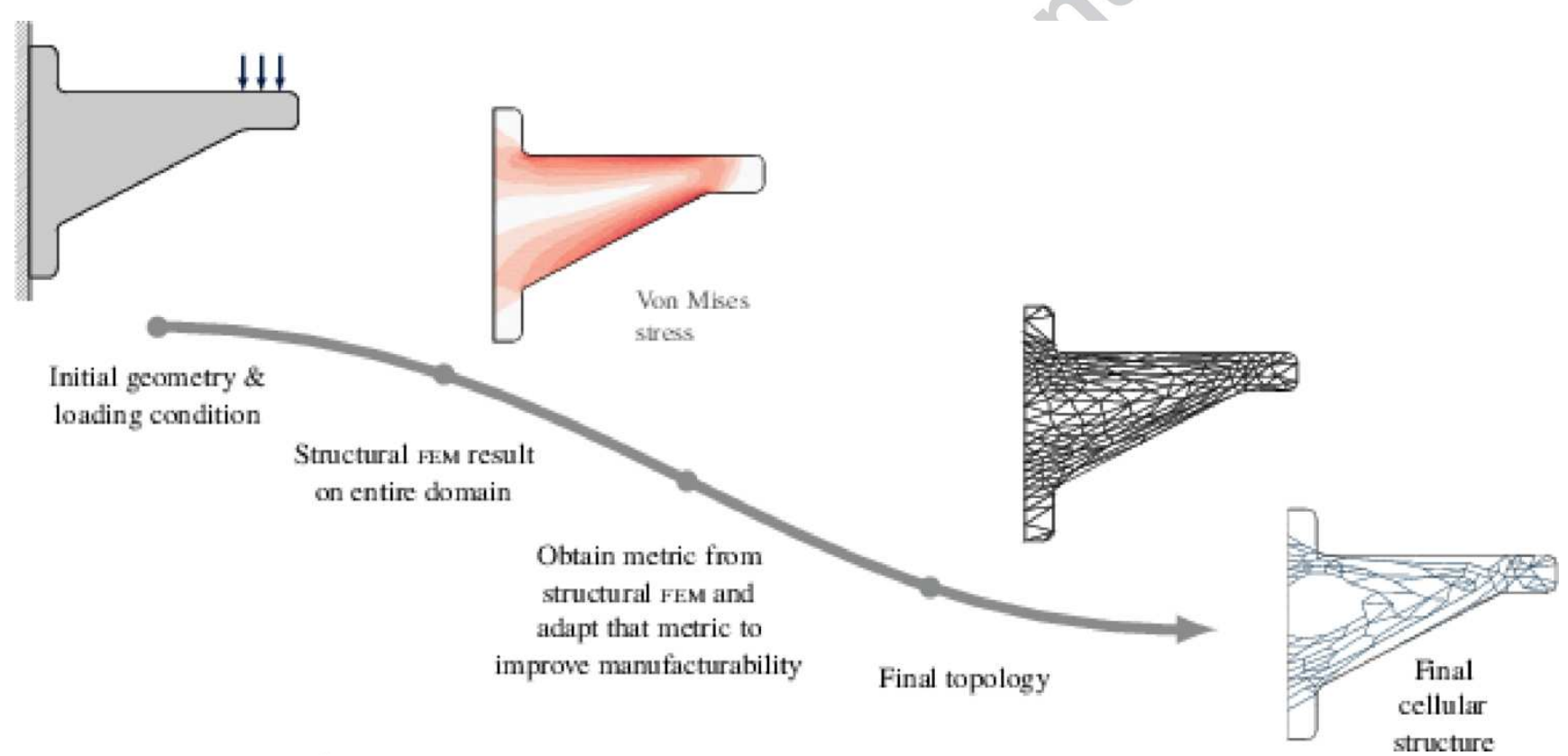

Fig. 2: Complete approach for designing a manufacturable load-dependent cellular structure.

mon approach is to find that printer orientation where the maximum number of struts are manufacturable and remove struts that do not comply with the manufacturing constraints (Shidid et al., 2016).

In this paper, we develop a methodology for designing optimal and manufacturable cellular structures to be manufactured using additive manufacturing. A major goal here is to reduce the computational burden of designing such cellular structures to allow for inclusion in early design stages, which is enabled by the use of low-order methods and adaptive meshing techniques. The design methodology is split in two main parts: (1) the design of the structural topology (Section 2), and (2) finding the optimal thickness of each strut (Section 3) - this approach is also illustrated in Fig. 2. This approach is not globally optimal, but allows for a large reduction in computational design effort. The designed cellular structure is then converted into a solid CAD model to allow for rendering and manufacturing (Section 4). The methodology is demonstrated for several 
brackets in Section 5 and concluding remarks are provided in Section 6.

\section{Design of Cellular Topology}

To design lightweight cellular structures, the cellular topology - i.e., the location of the nodes and the connectivity between those nodes - needs to be aligned with the load direction and needs to be fine in areas of high stress, while it can be coarser in locations of lower stress. Moreover, the cellular topology should be defined such that it is actually manufacturable.

In this work, a Riemannian metric field (Section 2.1) is used to define the cellular topology, where the metric is informed by the stress tensor throughout the available design space of the problem, which is found by solving a linear elasticity problem on a fully solid design domain (Section 2.2). The metric field is based on this stress tensor, subject to corrections for direction and positive definiteness (Section 2.3). Moreover, information about manufacturing constraints and the build direction can be included in the Riemannian metric (Section 2.4)

\subsection{Riemannian Metric Fields}

A cellular structure can be described from a discrete or continuous viewpoint. In the discrete viewpoint, the cellular structure consists of nodes and struts, each with a certain length, thickness, and direction. In the continuous viewpoint, the properties of the cellular structure such as the local size-i.e., the volume that each element of the cellular structure encompasses - and directionality of the cellular topology can be described as a continuous field throughout the structure, as shown in Fig. 3. The latter is a more elegant way of looking at the problem of designing a load-dependent cellular topology and also relaxes the intractability of solving a discrete optimization problem for the optimal topology. This continuous approach is demonstrated in the following.

The goal here is to design a load-dependent cellular topology - i.e., the location of the nodes and direction of the struts of the cellular topology are tailored to the specific loading condition(s) to which the part is subjected. To design such a load-dependent cellular topology, we leverage mesh adaptation techniques from the CFD community. In CFD applications, meshes need to be locally refined in areas with large gradients, such as boundary layers and shock waves. Furthermore, these are areas where the gradient in one direction is large, while being small in a perpendicular direction, requiring the mesh to exhibit a certain anisotropy. One way of prescribing anisotropy is through the notion of Riemannian metric spaces, which were first used by Diaz et al. (1997) and Hecht et al. (1997) for mesh adaptation purposes in fluid flow problems. Such a metric is a tensor field that at any point in the domain describes mesh size and anisotropy. Mesh adaptation using a metric computed from solution error is demonstrated by, amongst others, Loseille and Alauzet (2011a,b), and Yano and Darmofal (2012). Metric-based anistropic mesh adaptation has also been used to increase the efficiency of SIMP-based topology optimization methods (Ejlebjerg Jensen, 2016).

Most of these adaptive methods are developed for meshes with simplicial elements, which are also appropriate for structural applications. Triangles - or tetrahedra in three-dimensional structures - have good static structural properties and are therefore appropriate core elements for a cellular structure. Using such a metric to describe the triangulation is a natural way to include information of load paths into the cellular structure, and also to include manufacturing constraints, as we show in Section 2.4

We now present the formal mathematical definition of a Riemannian metric field, closely following the notation by Loseille and Alauzet (2011a) and Loseille and Alauzet (2011b). A Riemannian metric field $\{\mathcal{M}(\mathbf{x})\}_{\mathbf{x} \in \Omega}$ with $\mathbf{x}$ the physical coordinate - is a smoothly varying field of semi-positive definite (SPD) matrices on the $d$ dimensional domain $\Omega \subset \mathbb{R}^{d}$. The edge length of a segment $\mathbf{a b}$ from $\mathbf{a} \in \Omega$ to $\mathbf{b} \in \Omega$ under this Riemannian metric is given by

$\ell_{\mathcal{M}}(\mathbf{a b})=\int_{0}^{1} \sqrt{\mathbf{a} \mathbf{b}^{\top} \mathcal{M}(\mathbf{a}+\mathbf{a b} s) \mathbf{a b}} \mathrm{d} s$.

The metric-conforming triangulation $\mathcal{T}_{h}$ is then such that all edges are close to unit length under the Riemannian metric field, $\{\mathcal{M}(\mathbf{x})\}_{\mathbf{x} \in \Omega}$, typically satisfying $\frac{1}{\sqrt{2}} \leq \ell_{\mathcal{M}}(e) \leq \sqrt{2} \quad \forall e \in \operatorname{Edges}\left(\mathcal{T}_{h}\right)$

A geometric interpretation of a metric and its corresponding triangulation are illustrated in Fig. 4. As a further example of a metric field, consider the metric field in Fig. 5, which was used to generate the cellular structure in Fig. 3.

\subsection{Solid Mechanics Solver}

To find the continuous stress tensor, we solve the linear elasticity equations over the whole domain. The full 


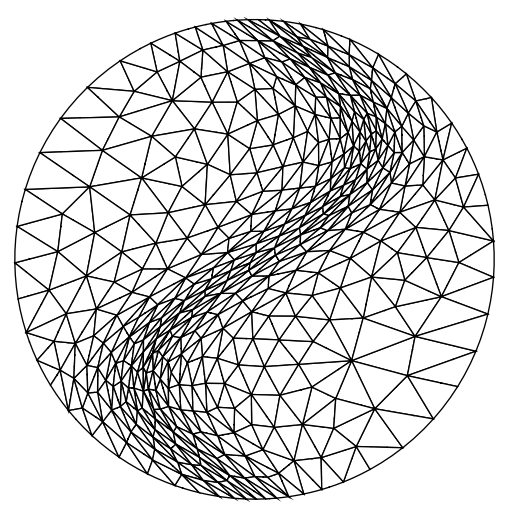

(a) Discrete cellular structure

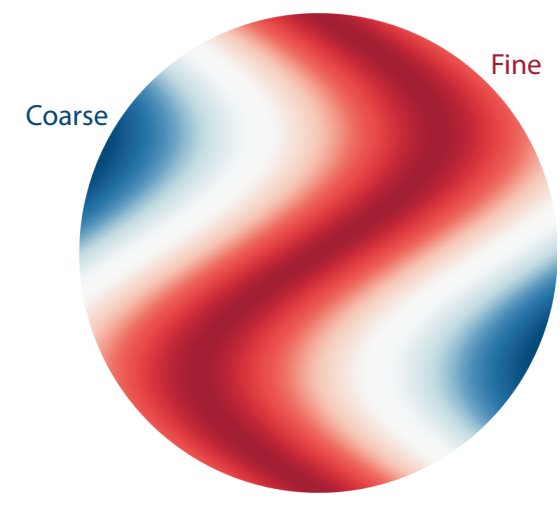

(b) Continuous field of local element size of cellular topology (based on determinant)

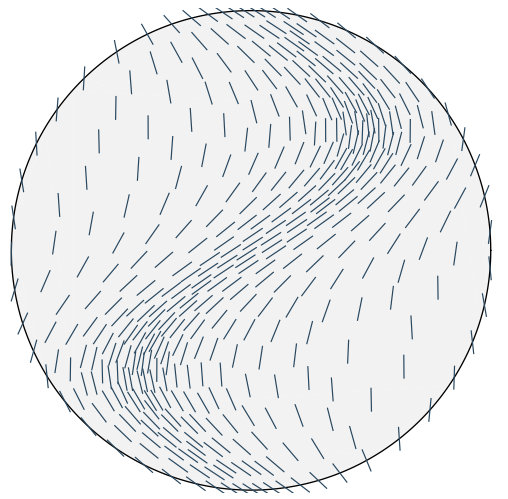

(c) Continuous field of local direction of cellular topology

Fig. 3: Cellular structure and continuous fields of its properties.

linear elasticity equations are (Shames and Pitarresi, 2000)

$$
\begin{aligned}
\nabla \cdot \underline{\boldsymbol{\sigma}}+\mathbf{f} & =0 & & \forall \mathbf{x} \in \Omega \\
\underline{\boldsymbol{\epsilon}} & =\frac{1}{2}\left[\nabla \mathbf{u}+(\nabla \mathbf{u})^{\top}\right] & & \forall \mathbf{x} \in \Omega \\
\underline{\boldsymbol{\sigma}} & =\underline{\boldsymbol{C}}: \underline{\boldsymbol{\epsilon}}, & & \forall \mathbf{x} \in \Omega \\
\mathbf{u} & =\overline{\mathbf{u}}, & & \forall \mathbf{x} \in \partial \Omega_{D} \\
\underline{\boldsymbol{\sigma}} \cdot \mathbf{n} & =\mathbf{t}, & & \forall \mathbf{x} \in \partial \Omega_{N}
\end{aligned}
$$

where $\Omega$ is the domain of the problem, $\boldsymbol{\sigma}$ is the Cauchy stress tensor, $\underline{\epsilon}$ is the infinitesimal strain tensor, $\mathbf{u}$ is the displacement vector, $\underline{\boldsymbol{C}}$ is the fourth-order stiffness tensor, and $\mathbf{f}$ is the body force per unit volume. As for the boundary conditions, $\overline{\mathbf{u}}$ is the specified displacement of a boundary $\partial \Omega_{D}$ (Dirichlet boundary condition), $\mathbf{t}$ is the specified stress normal to a boundary $\partial \Omega_{N}$ (Neumann boundary condition), $\mathbf{n}$ is a normal vector (positive outward). Note that the : operator indicates a double contraction, such that Eq. (1c) can be written as $\sigma_{i j k l}=C_{i j k l} \epsilon_{k l}$ in index notation. $\underline{\boldsymbol{C}}$ is defined as

$C_{i j k l}=\lambda_{1} \delta_{i j} \delta_{k l}+\mu_{s}\left(\delta_{i k} \delta_{j l}+\delta_{i l} \delta_{j k}\right)$,

where $\lambda_{1}$ is Lamé's first parameter, and $\mu_{s}$ is the shear modulus (or rigidity), both of which are elastic moduli, defined as

$\lambda_{1}=\frac{E \nu}{(1+\nu)(1-2 \nu)}$

$\mu_{s}=\frac{E}{2(1+\nu)}$

where $E$ is the Young's modulus, and $\nu$ is Poisson's ratio.

The linear elasticity equations are solved using a finite element method (FEM), specifically a hybridizable discontinuous Galerkin discretization, following the approach by Soon et al. (2009).

More details on that discretization and the solver are provided in Appendix B of (Opgenoord, 2018). ${ }^{2}$

\subsection{Load-Dependent Cellular Topology Based on}

\section{Metric Field}

To tailor the cellular topology to the load direction and areas of high stress, we base the topology on the stress tensor throughout the solid domain. The stress tensor $\underline{\boldsymbol{\sigma}}$ indicates which areas of the domain are highly loaded. Intuitively, it is desirable to have a finer cellular structure near highly loaded areas, and to have a coarser cellular structure near areas with low stress. Furthermore, the structure should align with the stress direction. Therefore, we adapt the structure to the stress tensor using metric-based mesh adaptation techniques (Diaz et al., 1997; Yano and Darmofal, 2012).

Our proposed method for computing the metric from the stress tensor applies corrections to ensure positive definiteness of the metric and to ensure that the direction of the metric aligns with the stress direction. The stress tensor is not necessarily positive definite because it distinguishes between compression and tension (Fig. 6), whereas a metric is required to be positive definite. For adaptation purposes, however, it is not important to distinguish between compressive and tensile stresses since it is the magnitude of the stresses that drives the need for local refinement. ${ }^{3}$ Therefore,

2 The source code for this solver has been made available at github.com/mopg/luteos.jl.

3 In designing the topology of the cellular structure, buckling is ignored. 


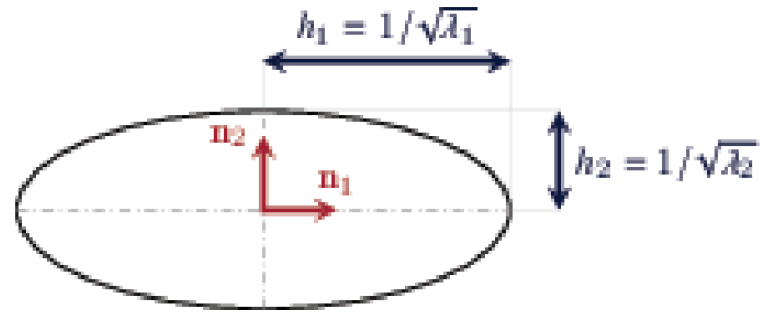

(a) Metric in $2 \mathrm{D}$

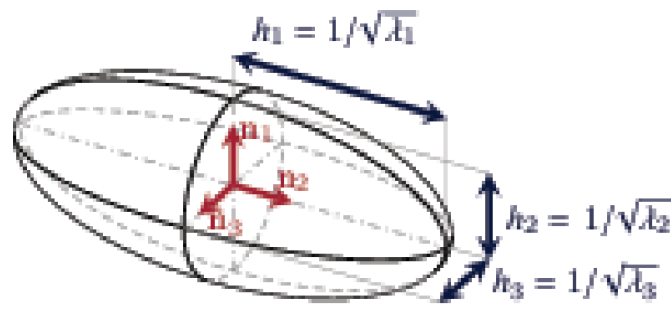

(b) Metric in 3D

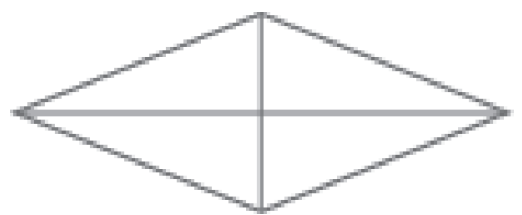

(c) Example triangulation in 2D conforming only to the metric defined at the center of ellipse

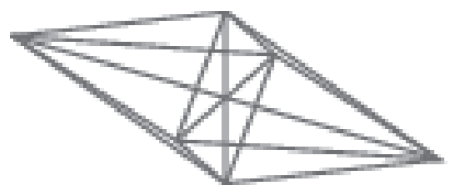

(d) Example triangulation in 3D conforming only to the metric defined at the center of ellipsoid

Fig. 4: Geometric interpretation of a metric. Note that the triangulation corresponding to the metric is not unique.

to guarantee the metric is positive definite, we take the magnitude of the principal stresses to be positive, such that this metric is positive definite -i.e., it has a positive determinant. In practice, this is implemented by taking the eigenvalue decomposition of the stress tensor and taking the absolute value of the eigenvalues to compute the metric--while ensuring the eigenvalues stay above a minimum threshold to avoid a semi-definite metric.

Secondly, to align the topology with the stress direction, the eigenvalues of the metric are altered. The magnitude of the local determinant of the metric is inversely proportional to the local size of the mesh. Therefore, when the determinant of the stress tensor is large in a particular part of the domain, the element sizes of the topology are small in that part as well, which is desired. If the stress is high in one particular direction,

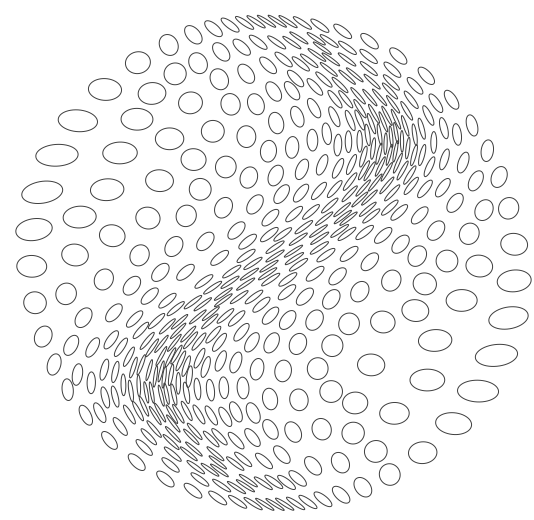

Fig. 5: A metric is a field of SPD tensors, illustrated using ellipses here. This metric field is used to generate the cellular structure in Fig. 3.

the local topology should be elongated along that direction, and thus the metric should be smaller in that direction. Therefore, the eigenvalues of the final metric are taken to be the inverse of the eigenvalues of the metric based on the stress tensor - while ensuring that the area under the metric stays constant. This is illustrated for two-dimensional problems in Fig. 7.

For three-dimensional problems, we take the inverse of the principal stresses while scaling the metric such that the determinant of the metric stays the same. For three-dimensional problems, the metric is therefore computed as

$m_{1}=\sqrt[3]{S^{2}} \frac{1}{\sigma_{1}}, \quad m_{2}=\sqrt[3]{S^{2}} \frac{1}{\sigma_{2}}, \quad m_{3}=\sqrt[3]{S^{2}} \frac{1}{\sigma_{3}}$,

where $S \equiv \sigma_{1} \sigma_{2} \sigma_{3}$ and $m_{1}, m_{2}$, and $m_{3}$ are the eigenvalues of the final metric $\mathcal{M}$. The metric $\mathcal{M}$ is used to define the topology.

While not implemented in this paper, this approach could extend to multiple load cases as well. In that case, the metric $\mathcal{M}$ could be based on a weighted combination of the stress fields corresponding to those load cases. The incorporation of manufacturing constraints into that metric will be the same as is described in the next subsection.

\subsection{Manufacturing Constraints}

Although additive manufacturing allows for large design freedom, few AM processes can manufacture any type of cellular structure. Most processes are for instance limited in terms of the overhang tolerated for parts. Typically, extrusion-based manufacturing processes have more stringent overhang constraints than powder-based manufacturing techniques, but almost all 


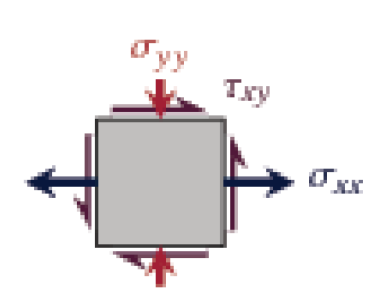

$\operatorname{det}\left(\begin{array}{cc}\sigma_{x x} & \tau_{M y} \\ \tau_{r y} & \sigma_{y y}\end{array}\right)<0$

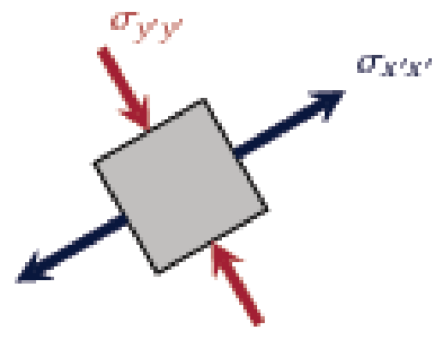

$\operatorname{det}\left(\begin{array}{cc}\sigma_{M^{\prime}} & 0 \\ 0 & \sigma_{y y}\end{array}\right)<0$

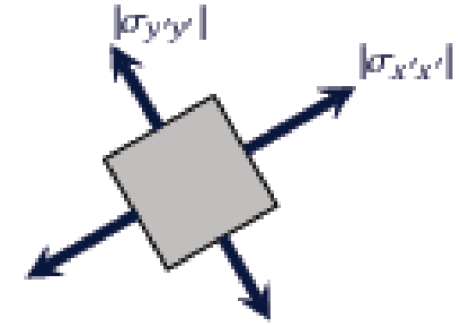

$\operatorname{det}\left(\begin{array}{cc}\left|\sigma_{x^{\prime}, r^{\prime}}\right| & 0 \\ 0 & \left|\sigma_{y y}\right|\end{array}\right)>0$

Fig. 6: The stress tensor is not necessarily positive definite, but an updated metric for adaptation purposes can be made positive definite by taking the absolute value of the principle stresses.
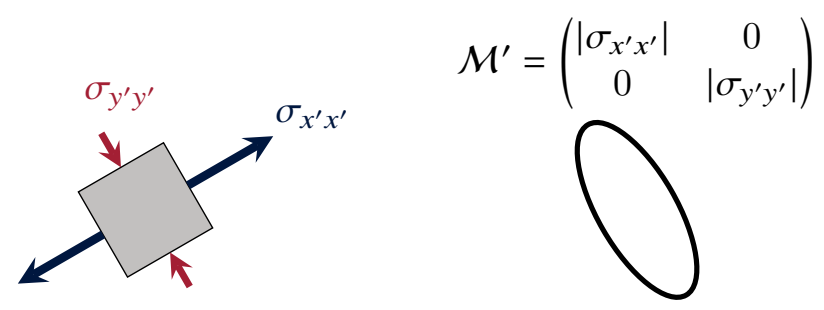

Example of simplex conforming to metric
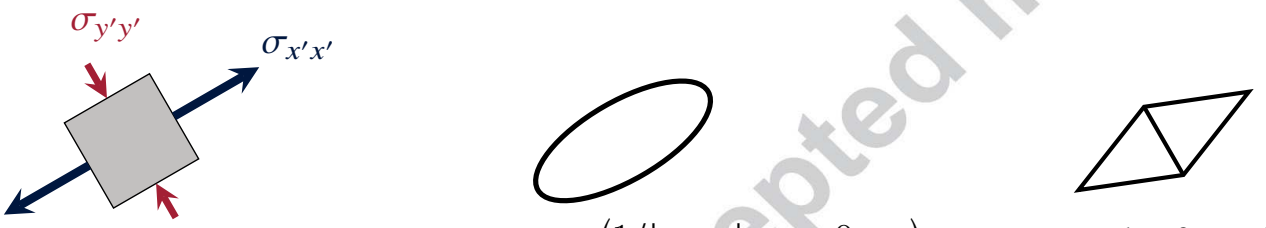

$$
\mathcal{M}^{\prime}=\left|\sigma_{x^{\prime} x^{\prime}} \sigma_{y^{\prime} y^{\prime}}\right|\left(\begin{array}{cc}
1 /\left|\sigma_{x^{\prime} x^{\prime}}\right| & 0 \\
0 & 1 /\left|\sigma_{y^{\prime} y^{\prime}}\right|
\end{array}\right)
$$

Example of simplex conforming to metric

Fig. 7: Using scaled eigenvalues for the final metric yields a topology with correct orientation. The top row illustrates what the topology would look like if the eigenvalues of the final metric were taken to be the absolute principal stresses, the bottom row illustrates the desired direction obtained by using scaled eigenvalues based on the principal stresses. $\mathcal{M}^{\prime}$ is the metric $\mathcal{M}$ expressed in the coordinate system of the principal stresses.

additive manufacturing processes have some form of overhang constraints.

The two most significant manufacturing constraints are the minimum geometric feature due to limited printer resolution, and the overhang angle constraint. The latter constraint is imposed because a new layer needs to have support from the structure below, as shown in Fig. 8. Even in powder-based manufacturing methods this constraint remains due to heat transfer and warping issues. We also include bridge constraints, which limit the maximum horizontal "bridge" that can be printed.

There are several different ways to include the manufacturing constraints. The minimum geometric feature size limit is straightforward to include by setting the minimum mesh size through the metric. For the over- hang angle constraint, we optimize the local metric such that it is as close as possible to the original metric in the Frobenius norm, while adhering to the manufacturing constraints. In order to solve that optimization problem, the orientation and shape of the metric needs to be described geometrically. Here, we choose quaternions (Hamilton, 1844; Wie, 2008) to describe the axis orientation of the metric, and the length of the major axes $\left(h_{1}, h_{2}, h_{3}\right)$ to describe the shape of the ellipsoid (Fig. 9). Quaternions are chosen over Euler or TaitBryan angles, because these have a singularity which can limit the convergence of the optimization algorithm. The optimization problem is described mathematically 


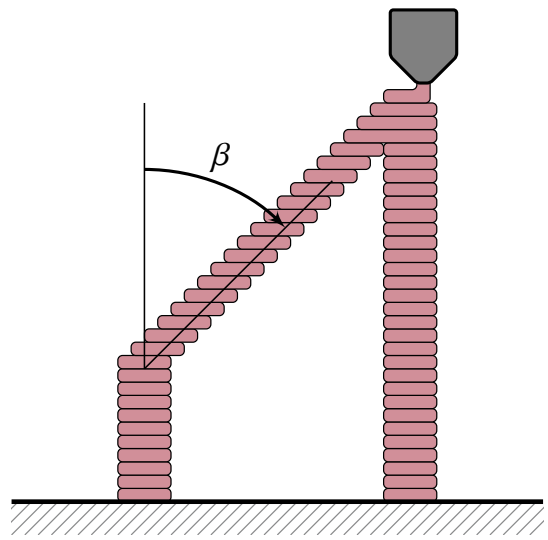

(a) Overhang angle

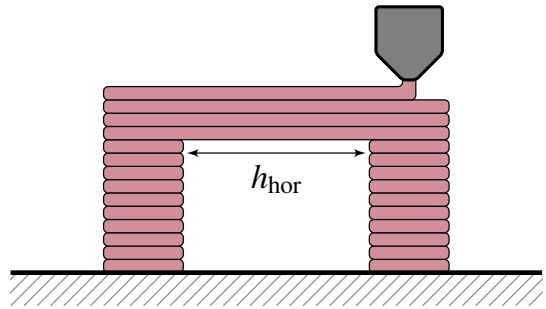

(b) Bridge length

Fig. 8: In this work, overhang angle constraints and bridge length constraints are considered.

as

$$
\min _{\mathbf{q}, h_{1}, h_{2}, h_{3}}\left\|\mathcal{M}-\mathcal{M}_{\text {org }}\right\|_{F}
$$

subject to $\frac{\mathbf{n}_{1} h_{1} \pm \mathbf{n}_{2} h_{2}}{\left\|\mathbf{n}_{1} h_{1} \pm \mathbf{n}_{2} h_{2}\right\|} \cdot \mathbf{n}_{\text {printer }} \geq \cos \beta_{\max }$

$$
\frac{\mathbf{n}_{1} h_{1} \pm \mathbf{n}_{3} h_{3}}{\left\|\mathbf{n}_{1} h_{1} \pm \mathbf{n}_{3} h_{3}\right\|} \cdot \mathbf{n}_{\text {printer }} \geq \cos \beta_{\max }
$$$$
\|\mathbf{q}\|=1
$$

where $\mathbf{q}=\left[q_{1}, q_{2}, q_{3}, q_{4}\right]^{\top}$ are the quaternions, and $\mathcal{M}=$ $\boldsymbol{R} \boldsymbol{\Lambda} \boldsymbol{R}^{\top}$ with $\boldsymbol{R}$ obtained from $\mathbf{q}$ as

$\boldsymbol{R}=\left[\begin{array}{ccc}1-2 q_{3}^{2}-2 q_{4}^{2} & 2 q_{2} q_{3}-2 q_{1} q_{4} & 2 q_{2} q_{4}+2 q_{1} q_{3} \\ 2 q_{2} q_{3}+2 q_{1} q_{4} & 1-2 q_{2}^{2}-2 q_{4}^{2} & 2 q_{3} q_{4}-2 q_{1} q_{2} \\ 2 q_{2} q_{4}-2 q_{1} q_{3} & 2 q_{3} q_{4}+2 q_{1} q_{2} & 1-2 q_{2}^{2}-2 q_{3}^{2}\end{array}\right]$

and $\boldsymbol{\Lambda}$ the eigenvalue matrix obtained from $h_{1}, h_{2}, h_{3}$ as

$\boldsymbol{\Lambda}=\left[\begin{array}{ccc}1 / h_{1}^{2} & 0 & 0 \\ 0 & 1 / h_{2}^{2} & 0 \\ 0 & 0 & 1 / h_{3}^{2}\end{array}\right]$

The normal vectors of the major axes of the ellipse$\mathbf{n}_{1}, \mathbf{n}_{2}, \mathbf{n}_{3}$-are the columns of $\boldsymbol{R} . \mathbf{n}_{\text {printer }}$ is the $z$ axis of the printer expressed in the coordinate system of the part. Note that this optimization problem is non-convex, due to the $\|\mathbf{q}\|=1$ constraint. A general nonlinear optimization solver is therefore used to solve this problem, in this case an interior point optimizer, Ipopt (Wächter and Biegler, 2006). A geometric

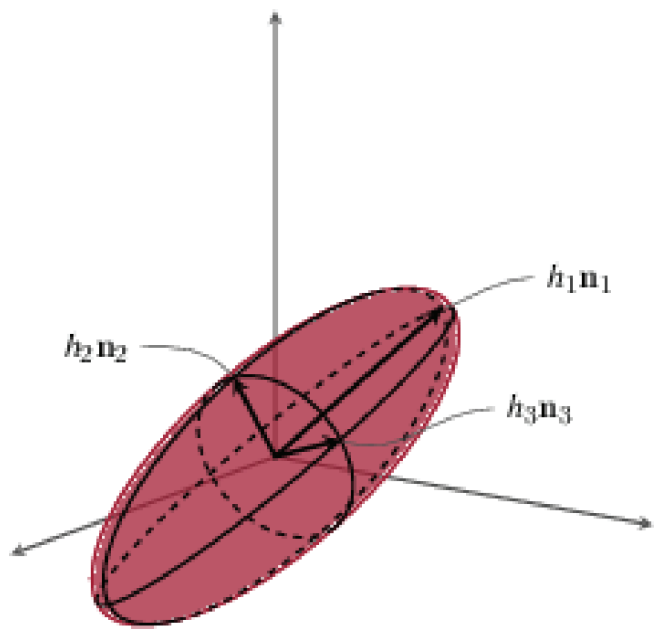

(a) Metric, defined by $h_{1}, h_{2}, h_{3}$ as well as $\mathbf{q}$ (from which $\mathbf{n}_{1}, \mathbf{n}_{2}, \mathbf{n}_{3}$ follow).

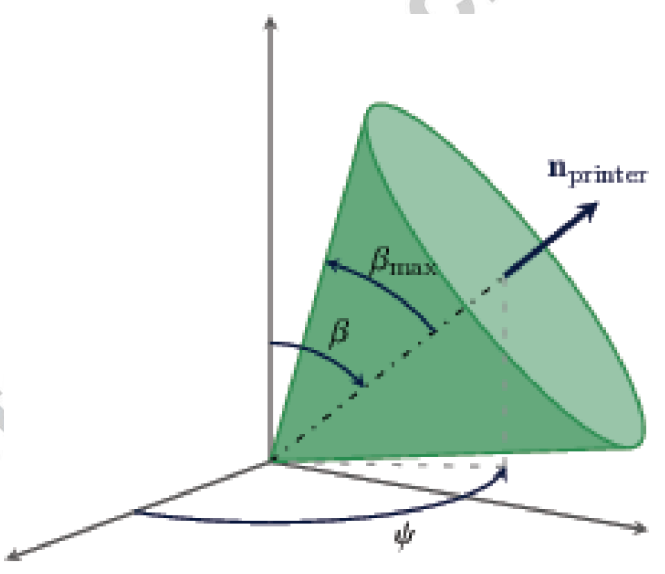

(b) Overhang constraints can be thought of as a cone in the direction of the printer's $z$-axis.

Fig. 9: Geometry of optimization problem for including manufacturing constraints in the local metric.

interpretation of this optimization problem is that the metric has to be oriented such that it points into the manufacturable cone (Fig. 9), while deviating as little as possible from the initial metric.

This approach to include manufacturing constraints into the metric is purely geometric, without a direct relationship to the loads through the cellular structure. An example of the result of the optimization in $2 \mathrm{D}$ is illustrated in Fig. 10, where in this case the metric is mostly rotated. However, even though this is a geometric approach, the impact on the load bearing capability of the structure is typically limited, since we find at each point in the domain a metric that is closest to the original metric obtained from the stress tensor, which ensures the topology is still as closely aligned 

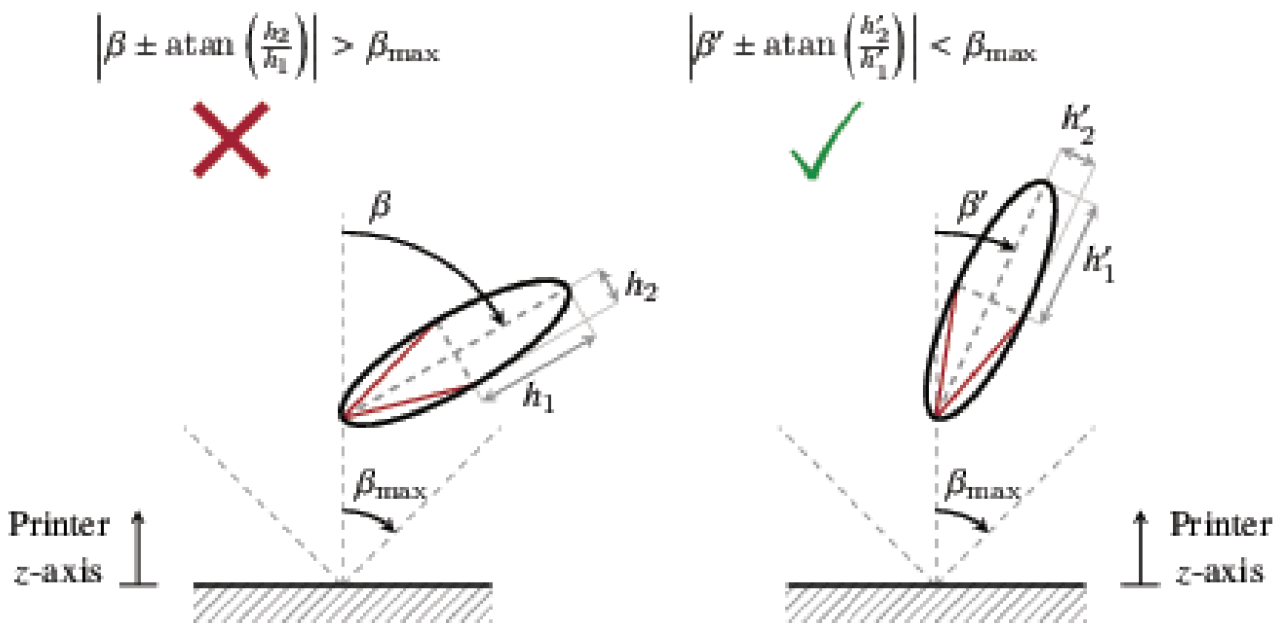

Fig. 10: 2D example of including manufacturing constraints by optimizing the local metric.

with the stress direction as possible. We demonstrate this by showing the relative specific compliance of a cellular structure - column under compression - when we change the build direction. For this cellular structure the cross-sectional areas for the struts is constant throughout the structure. We see that the specific compliance is fairly constant for small $\beta$ angles, but even for large printer angles $\left(40^{\circ}\right)$ the specific compliance is only $20 \%$ higher. We show the influence of printer angle on the direction of the cellular topology of this column in compression in Fig. 12, which clearly shows that the topology is oriented along the printer $z$-axis. Of course, when the build direction is too different from the load direction, the load-bearing capability of the structure will suffer more. The build direction should therefore be aligned as much as possible with the main load directions in the structure. A natural extension of this work would be to wrap an optimizer around our design methodology to find the best build direction, as is for instance done by Langelaar (2016).

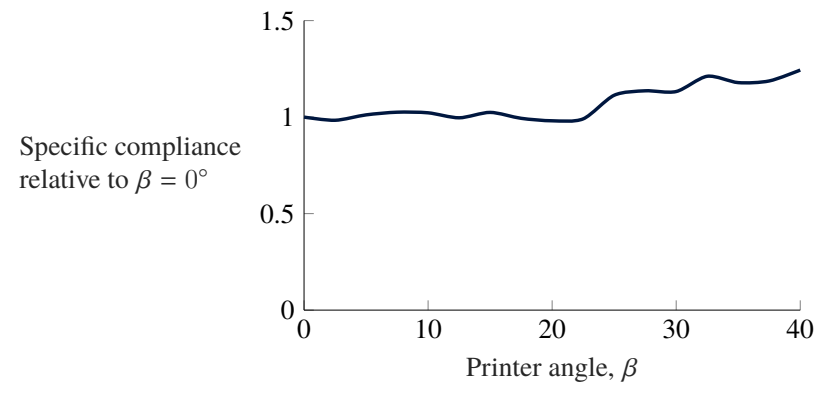

Fig. 11: Influence of printer angle on compliance of column in compression (same column as in Fig. 12).

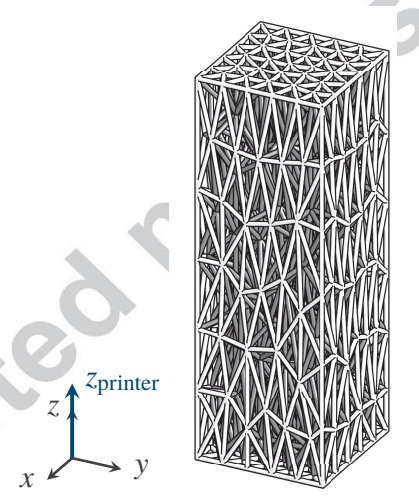

(a) $\beta=0^{\circ}$

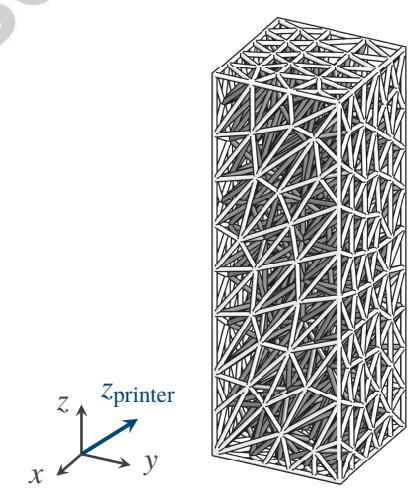

(b) $\beta=60^{\circ}$
Fig. 12: The cellular structure is aligned with the printer direction for this column under compression.

Once the metric is optimized to include manufacturing constraints, the topology of the cellular structure is generated using a metric-based mesh generator. Throughout this work, we use feflo. a by (Loseille and Lohner, 2010; Loseille, 2014; Loseille et al., 2017). To generate the initial mesh for which we compute the stress tensor, any mesh generator can be used, in this work typically commercial mesh generation software is used.

Even though the cellular topology is now tailored to the loads and aware of manufacturing constraints through the metric, not all struts may follow manufacturable directions. Several heuristic approaches can be taken to alter the cellular structure to ensure $100 \%$ manufacturability. One could find struts in the structure that violate the manufacturing constraints, and then perform edge swaps on them which in many cases may make the new strut manufacturable, see Fig. 13. 
Such edge swaps are commonly performed in meshing algorithms. For a 3D structure, however, we only perform these edge swaps on boundaries, as in the interior swapping edges may result in struts colliding with one another.

For struts that are perpendicular to the printer's $z$-axis, we need to ensure that they have enough support from below. In this work, we support the "horizontal" strut by finding a node under the strut and then adding struts between that node and the horizontal strut (Fig. 13).

The remaining struts that are not manufacturable could simply be removed, as is demonstrated by Shidid et al. (2016), but that does not necessarily guarantee manufacturability - one could remove a strut that was critical to support another strut-nor is there any guarantee that the resulting structure is still stable. For now, we take Shidid's approach, but a more integral approach is a topic of future work. In case the resulting structure is unstable, the overall density of the cellular structure can be altered or the build direction can be changed.

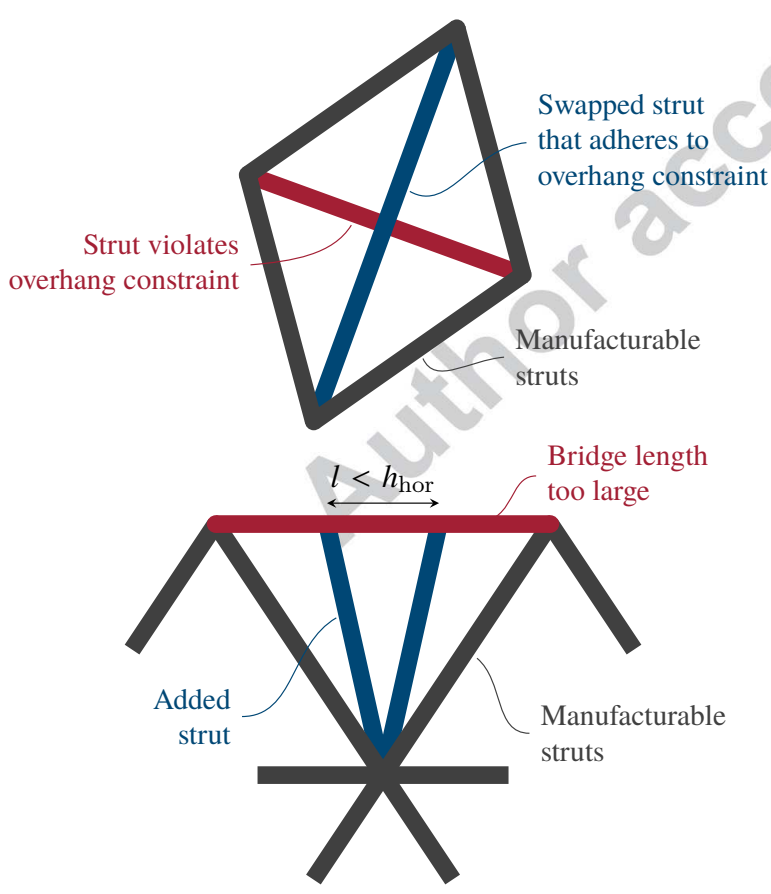

Fig. 13: The manufacturability of the cellular topology can be improved through edge swaps and by adding internal support.

\section{Optimal Cross-Sectional Area of Struts}

Once the cellular topology is defined, we can apply truss optimization techniques to optimize the size of each strut in that cellular structure. We therefore use pinned-joint theory to relate nodal loads to the strut loads and stresses, thereby assuming uniaxial loading of the struts and stresses in the nodes. This is a simplification that is made to reduce the computational effort for this early-stage design method. In later design stages, the struts themselves can be allowed to take up bending loads (using frame analysis) while also including the stresses in the nodes.

The truss optimization problem for the minimumweight solution with the cross-sectional area of the struts as design variables, can be cast into a linear programming form. Take $\mathbf{a}$ and $\mathbf{l}$ to be the vectors containing the areas and lengths, respectively, of the struts. Then, for a cellular structure with $n$ nodes and $m$ struts in physical dimension $d$, we minimize the volume of the cellular structure $(\mathcal{V})$ by solving (Kirsch, 1993)

$$
\begin{aligned}
\min _{\mathbf{a}, \mathbf{f}} \mathcal{V} & =\mathbf{l}^{\top} \mathbf{a} \\
\text { subject to } \boldsymbol{C} \mathbf{f} & =\mathbf{F} \\
-\sigma_{C} a_{j} & \leq f_{j} \leq \sigma_{T} a_{j} \\
a_{j} & \geq 0 \\
f & \in \mathbb{R}^{m}
\end{aligned}
$$

where $\mathbf{f}$ is a vector containing the forces in the struts, and $\mathbf{F}$ are the nodal forces - the definitions of these variables is also illustrated in Fig. 14. $\sigma_{C}$ and $\sigma_{T}$ are

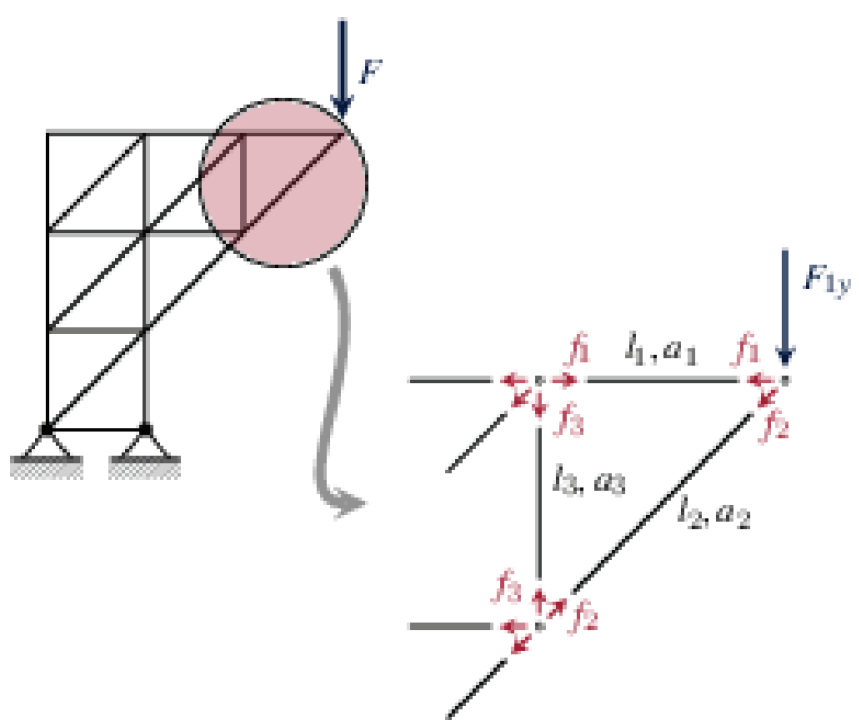

Fig. 14: A truss structure optimization is performed to minimize the weight of the cellular structure. 
the compressive and tensile stress limits, respectively. $\boldsymbol{C} \in \mathbb{R}^{n d \times m}$ is the connectivity matrix between nodes and struts, which is directly determined by the topology of the cellular structure. Each column of $\boldsymbol{C}$ is the projection of a strut on the degrees of freedom of the nodes that are connected to that strut. Therefore, the matrix entry corresponding to the $i$ th node and $j$ th strut for a three-dimensional cellular structure is expressed as

$C_{3(i-1)+1, j}=\mathbf{n}_{j} \cdot \hat{\mathbf{x}}$

$C_{3(i-1)+2, j}=\mathbf{n}_{j} \cdot \hat{\mathbf{y}}$

$C_{3(i-1)+3, j}=\mathbf{n}_{j} \cdot \hat{\mathbf{z}}$,

where $\hat{\mathbf{x}}, \hat{\mathbf{y}}$, and $\hat{\mathbf{z}}$ are the unit vectors in the $x, y$, and $z$ directions, respectively. As an example, consider Fig. 15 where strut $k$ is orientated in the $x y$-plane and makes a $60^{\circ}$ angle with the $x z$-plane.

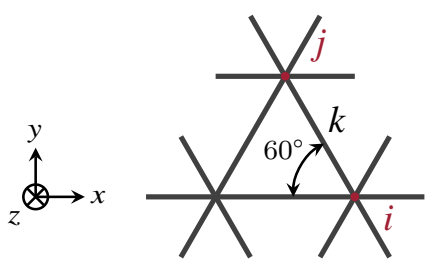

$$
\begin{aligned}
& C_{3(i-1)+1, k}=-\frac{1}{2} \\
& C_{3(i-1)+2, k}=\frac{1}{2} \sqrt{3} \\
& C_{3(j-1)+1, k}=\frac{1}{2} \\
& C_{3(j-1)+2, k}=-\frac{1}{2} \sqrt{3}
\end{aligned}
$$

Fig. 15: The matrix $\boldsymbol{C}$ defines the connectivity between the struts and nodes of the cellular structure.

This plastic formulation in Eq. (3) can be reformatted as a standard linear program and can therefore be solved very quickly. This formulation, however, can only be used for single load cases with no minimum area constraints, because the solution has to be a statically determinate structure, seeing as no stress-strain compatibility conditions are taken into account (Kicher, 1966). However, we require constraints to be placed on the cross-sectional areas of the struts. Therefore, a formulation which satisfies the stress-strain relations has to be used (Ohsaki, 2011)

$$
\begin{aligned}
\min _{\mathbf{a}, \mathbf{u}, \mathbf{f}, \boldsymbol{\sigma}} \quad \mathcal{V} & =\mathbf{l}^{\top} \mathbf{a} \\
\text { subject to } \quad \boldsymbol{C} \mathbf{f} & =\mathbf{F} \\
\boldsymbol{B} \boldsymbol{\sigma}+\boldsymbol{C}^{\top} \mathbf{u} & =\mathbf{0} \\
f_{j} & =\sigma_{j} a_{j} \\
-\sigma_{C} & \leq \sigma_{j} \leq \sigma_{T} \\
a_{\min , j} & \leq a_{j} \leq a_{\max , j} \\
\mathbf{u} & \in \mathbb{R}^{n d}, \mathbf{f} \in \mathbb{R}^{m}
\end{aligned}
$$

where $\boldsymbol{B} \in \mathbb{R}^{m \times m}$ is a diagonal matrix where the $j$ th diagonal entry corresponds to the deformation per unit force of the $j$ th strut,

$B_{j j}=\frac{l_{j}}{E a_{j}}$,

where $l_{j}$ and $a_{j}$ are the length and cross-sectional area of the $j$ th strut, respectively, and $E$ is the Young's modulus. This elastic formulation has been known to suffer from problems with vanishing constraints as $a_{j} \rightarrow 0$, resulting in $\sigma_{j}$ becoming independent of $f_{j}$ (Rozvany, 2001). Fortunately, we specifically want to prescribe a minimum area for each strut to ensure support for each strut, and therefore no vanishing constraints occur in this problem. Finally, note that we ignore self-weight in this optimization, i.e., we assume $f=0$ in Eq. (1).

The optimization problem in Eq. (4) can be extended to include buckling constraints. The critical Euler buckling load $f_{\text {crit }}$ of the $j$ th strut can be expressed as

$f_{\text {crit }, j}=\frac{\pi E a_{j}^{2}}{4\left(k_{e} l_{j}\right)^{2}}$,

where $k_{e}$ is the column effective length factor. This constraint is also nonlinear in $a_{j}$, which further adds to the nonlinearity of Eq. (4). This constraint is added to the optimization statement in Eq. (4) as

$f_{j} \geq-f_{\text {crit }, j}$.

Multiple load cases can also be included in the strut diameter optimization problem (4) by adding additional force-balance, stress-strain compatibility, stress definition, and stress limit constraints, as well as additional displacement, force, and stress optimization variables corresponding to each load case.

As written, Eq. (4) is not convex, due to the $f_{j}=$ $\sigma_{j} a_{j}$ constraint. When the minimum compliance problem is solved instead - maximizing stiffness, rather than minimizing weight - the design problem can be written as a convex optimization problem (Freund, 2004). However, we specifically require the minimum-weight solution, which results in a non-convex optimization problem. The problem is therefore solved as a generic nonlinear optimization problem using Ipopt (Wächter and Biegler, 2006). 


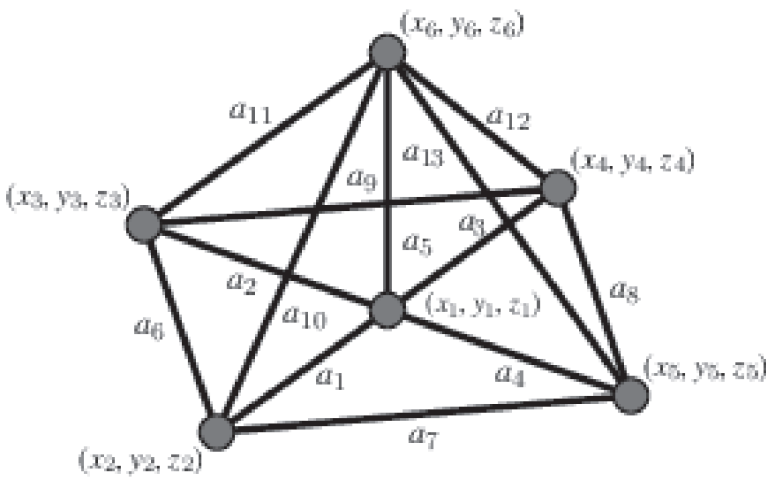

Fig. 16: Cellular structure representation with 6 nodes and 13 struts.

\section{Geometry Generation for Cellular Structures}

After the cellular topology is designed and the optimal cross-sectional area for each strut in the cellular structure is found, the design of the cellular structure is essentially a list of nodes coordinates and a list of cross-sectional areas of struts connecting those nodes (Fig. 16). This representation is a cheap representation of a complicated model, and is ideal to use in designing the part. However, in order to prototype a part, this information has to be translated into a solid representation of the part (Fig. 17). Note that fillets in the joints are not taken into account in this geometry generation algorithm. Such fillets, however, are crucial to avoid large stress concentrations in the nodes of the cellular structure.

We have developed a geometry generation algorithm that can quickly transform a large heterogeneous cellular structure representation into a watertight solid representation (in either .stl format or a parametric CAD format). ${ }^{4}$ We consider a heterogeneous cellular structure to be a cellular structure without repeating elements (element density varies over the structure and is dependent on the direction), and consider a homogeneous cellular structure to be a structure with repeating elements. In standard mesh terminology, a heterogeneous structure can also be referred to as anisotropic, and a homogeneous one as isotropic. McMillan et al. (2015) explored similar ideas for geometry generation, but their algorithm is restricted to using period unit cells, whereas we specifically require an algorithm for heterogeneous cellular structures. Our geometry generation algorithm is described in detail in Appendix $\mathrm{C}$ of (Opgenoord, 2018).

\footnotetext{
4 The source code for the geometry generation algorithm is made available at github.com/mopg/intrico.jl.
}

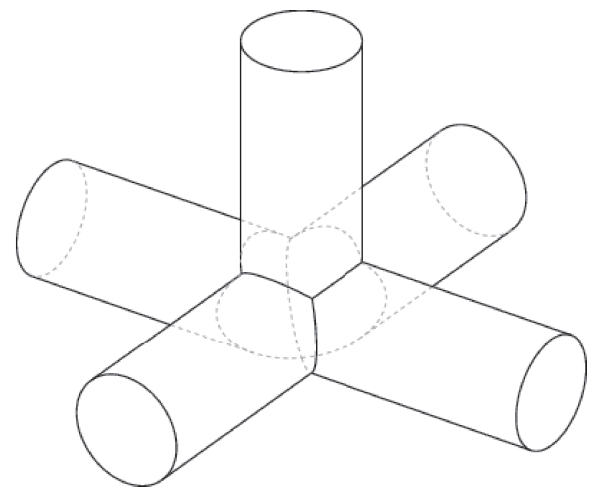

Fig. 17: Solid geometry for node 1 of Fig. 16.

\section{Example Designs}

The design methodology in Sections 2 and 3 is demonstrated here for several test cases. A simple bracket is designed in Section 5.1, which is used as a test case to compare the performance of a homogeneous cellular structure to that of a heterogeneous cellular structure and to describe the influence of the strut crosssectional area optimization on the mass of a part. We further demonstrate the design methodology on a test case from General Electric in Section 5.2, which allows us to compare our methodology to methods from the literature.

\subsection{Generic Bracket}

As a first example to demonstrate our design methodology, we design a generic bracket, for which the load case is shown in Fig. 18. In the following, we compare the performance of a tailored cellular topology to that of a homogeneous cellular topology and also characterize the influence of printer angle on the manufacturability of the part. We consider the design domain and load case shown in Fig. 18. The part is to be manufactured using standard ABS plastic. The Von Mises stress throughout the part is shown in Fig. 19 for that load case. As expected the Von Mises stress is highest near the "neck" of the structure, both because that part has the lowest cross-sectional area and because of the discontinuity in the geometry of the bracket, which leads to stress concentrations.

From the stress tensor computed for the load case in Fig. 18, we compute a metric field. The cellular topology is then generated using a metric-based mesh generator, in this case feflo.a (Loseille and Lohner, 2010). The cross-sectional area of the struts in that resulting cellular are optimized to yield the minimum-weight structure, subject to stress and buckling constraints. In 

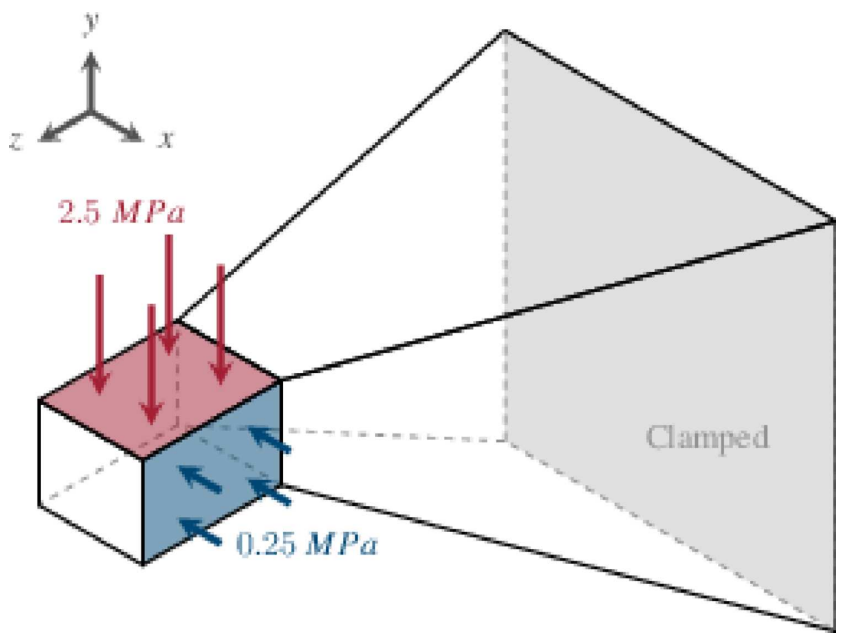

Fig. 18: Load case for bracket design example. The largest dimension is in $z$-direction $(150 \mathrm{~mm})$.

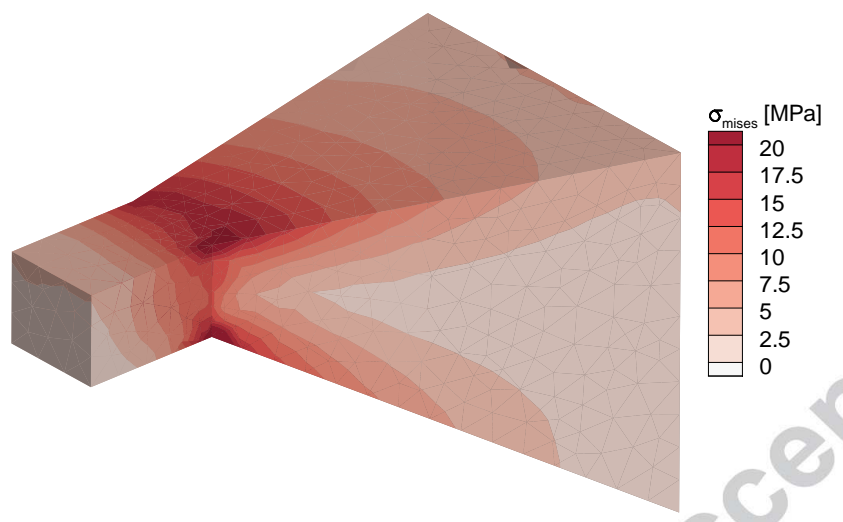

Fig. 19: Von Mises stress through domain of bracket for load case in Fig. 18.

the optimization we limit the diameter of the struts to be between $3 \mathrm{~mm}$ and $5 \mathrm{~mm}$. This lower limit is imposed since the bracket is to be manufactured using Fused Filament Fabrication (FFF), which requires a minimum strut diameter of around $3 \mathrm{~mm}$ (Redwood et al., 2017). Under these constraints, the resulting optimized part weighs only $0.140 \mathrm{~kg}$ for a cellular structure with 401 struts. A render of this part is shown in Fig. 20.

To investigate the importance of the cellular topology, we compare the minimum weight of the heterogeneous cellular structure - which is aligned with stress directions - to the minimum weight of a homogeneous cellular structure which is not tailored to the stress directions. For fair comparison, the heterogeneous and homogeneous cellular structures have the same number of struts. For the homogeneous cellular structure then, we also optimize the cross-sectional area of each strut. For the optimization problem of the homogeneous cellular structure, the diameter constraints of the

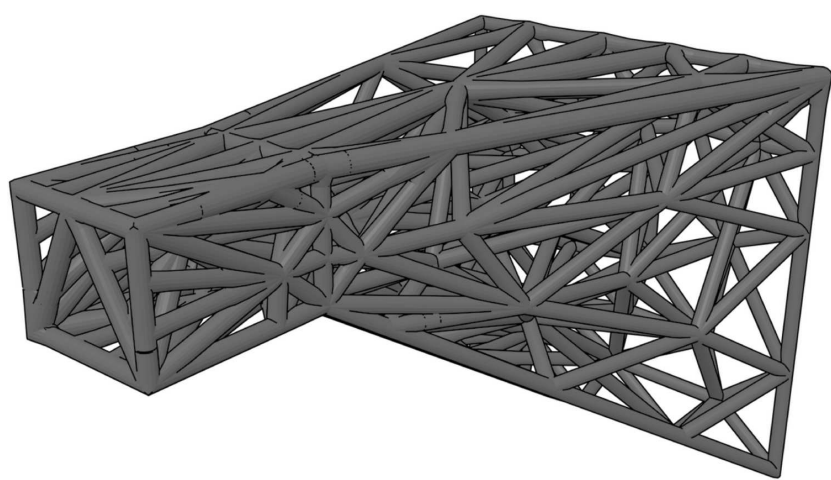

(a) Heterogeneous cellular structure

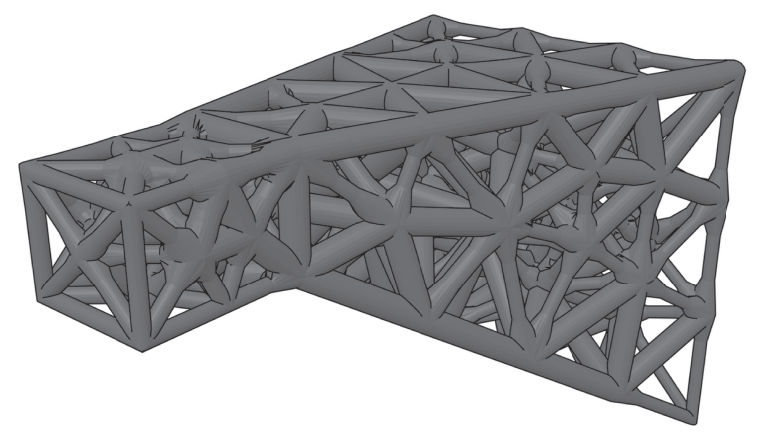

(b) Homogeneous cellular structure

Fig. 20: Comparison of optimized heterogeneous cellular structure and optimized homogeneous cellular structure.

struts have to be relaxed slightly compared to the heterogeneous cellular structure to ensure a feasible design. The diameter of the struts is therefore constrained to be between $3 \mathrm{~mm}$ and $6 \mathrm{~mm}$. The optimized homogeneous cellular structure weighs $0.209 \mathrm{~kg}-\mathrm{a} 37 \%$ increase compared to the heterogeneous cellular structure. These designs are also compared in Fig. 20. We see in Fig. 20 that the homogeneous cellular structure has a few thick struts while most struts are assigned the minimum strut diameter. This could be expected, because there are only few struts that are aligned with the stress direction and therefore there are only a few struts that take up most of the load. The heterogeneous cellular structure is therefore also expected to exhibit better load-bearing capability in case one or more struts fail. Also note that the manufacturability of the homogeneous cellular structure is only $59 \%$, whereas for the heterogeneous cellular structure it is $100 \%$-this again is expected, because the heterogeneous cellular structure is actually designed with manufacturing constraints in mind. To demonstrate the manufacturability of the heterogeneous cellular structure, the bracket 
is manufactured using an FFF desktop printer - the printed part is shown in Fig. 21.

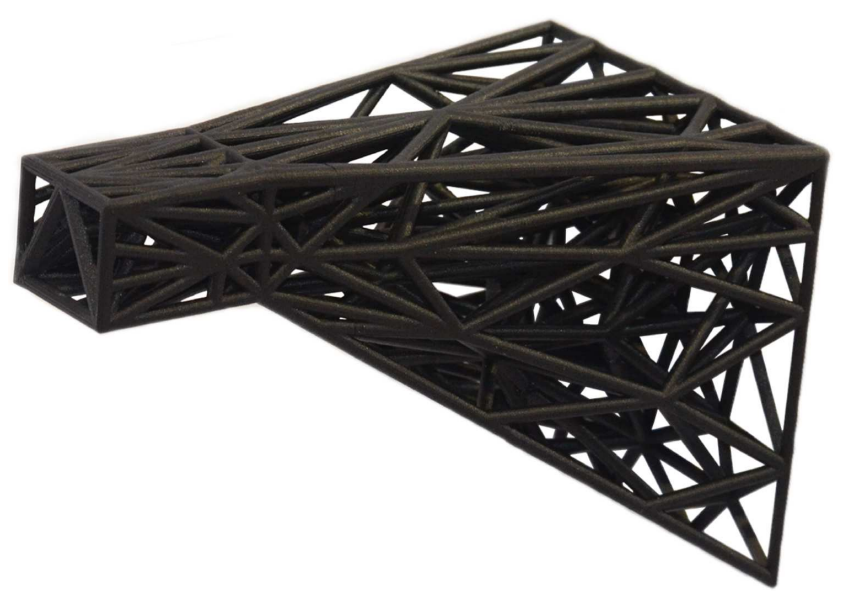

Fig. 21: This heterogeneous cellular structure is manufactured using the Markforged Mark II desktop printer.

As a final comparison, we investigate the influence of optimizing the cross-sectional area of each strut in the cellular structure on the performance of a part. We compare the previous designs to cellular structures with a uniform cross-sectional area for all struts for both the heterogeneous and homogeneous cellular structures; we call these designs uniform cellular structures. The minimum-weight uniform cross-sectional area for the uniform cellular structures is found by a bisection method, which finds the minimum cross-sectional area for which one or more struts are at the stress limit. We compare these designs in Table 1, where we see that the uniform cellular structures are at least $51 \%$ heavier than their non-uniform counterparts.

\begin{tabular}{lrc}
\hline & Mass, $\mathrm{kg}$ & Manufacturability, \% \\
\hline Heterogeneous & 0.152 & 100 \\
$\begin{array}{c}\text { Heterogeneous } \\
\quad \text { (uniform) }\end{array}$ & 0.230 & 100 \\
Homogeneous & 0.232 & 59.2 \\
$\begin{array}{c}\text { Homogeneous } \\
\quad \text { (uniform) }\end{array}$ & 0.367 & 59.2 \\
\hline
\end{tabular}

Table 1: Manufacturability and performance for heterogeneous and homogeneous cellular structures.

Finally, we also compare the influence of the design for manufacturability on the actual manufacturability of the resulting cellular structure. For this comparison, we design a cellular structure for various manufacturing directions by varying $\beta$ for the design and then check the manufacturability of the part under different $\beta$ manufacturing angles. Manufacturability here is defined as the ratio between the number of struts that follow manufacturable directions to the total number of struts in the part. The results in Table 2 show that whenever the part is to be manufactured in the same direction as it is designed, the manufacturability is $100 \%$, validating the approach. However, when we vary the angle at which the part is manufactured, the manufacturability quickly drops to levels as low as $53 \%$. Incorporating manufacturing constraints for the cellular structure into the design is therefore crucial.

Not only can we include critical design constraints early, the algorithm is fast as well, enabling inclusion in early design phases. Generating the inhomogeneous heterogeneous cellular structure only takes 28.5 seconds $^{5}$ to go from outer geometry definition and loading condition to a generated .stl file.

\begin{tabular}{rc|ccccc} 
& \multicolumn{6}{c}{$\beta$ (manufacturing) } \\
\cline { 2 - 7 } & in $\%$ & $-45^{\circ}$ & $-25^{\circ}$ & $0^{\circ}$ & $25^{\circ}$ & $45^{\circ}$ \\
\cline { 2 - 7 } & $-45^{\circ}$ & $\mathbf{1 0 0}$ & 97.1 & 99.3 & 87.7 & 60.0 \\
(design) & $-25^{\circ}$ & 77.5 & $\mathbf{1 0 0}$ & 98.6 & 94.2 & 67.8 \\
$0^{\circ}$ & 78.5 & 97.2 & $\mathbf{1 0 0}$ & 94.9 & 74.1 \\
& $25^{\circ}$ & 72.9 & 94.7 & 98.7 & $\mathbf{1 0 0}$ & 78.3 \\
& $45^{\circ}$ & 53.4 & 86.5 & 98.8 & 96.6 & $\mathbf{1 0 0}$ \\
\hline
\end{tabular}

Table 2: Manufacturability (\%) for manufacturing and designing a bracket with different manufacturing orientations.

\subsection{Aircraft Engine Bracket}

In 2013, General Electric (GE) launched a competition to redesign an aircraft engine bracket to take advantage of additive manufacturing capabilities. Although the deadline for the competition has long since passed, this design problem still serves as a useful test case for this work. Tang et al. (2015) used this bracket case study to demonstrate their lattice optimization algorithm, the result of which we compare our methodology against. Note that in this study only one load case is used - a $42.5 k N$ bearing load, see Fig. 22. This bracket is to be manufactured using Titanium Ti- $6 \mathrm{Al}-4 \mathrm{v}$, which has a tensile yield stress of $880 M P a$, a compressive yield stress of $970 M P a$, and a Young's modulus of 113.8 GPa.

528.5 seconds on one core of a 2015 Macbook Pro $(3.1 \mathrm{GHz}$ Intel Core i7). Note that this is including solving the FEM problem, which takes 15.5 seconds. 


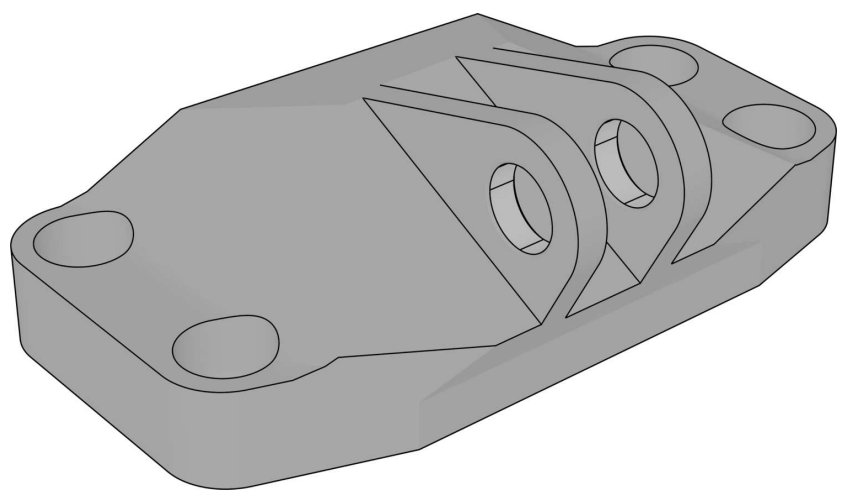

(a) Geometry of GE bracket.

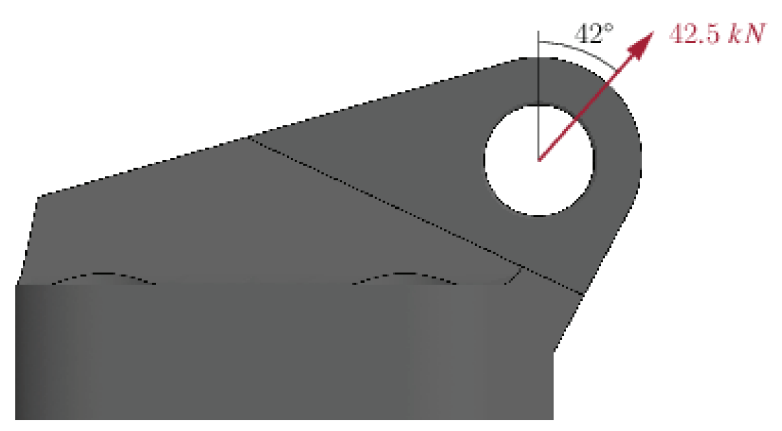

(b) Load case for GE bracket.

Fig. 22: The load case for the GE aircraft bracket is a $42.5 k N$ force applied at a $42^{\circ}$ angle from vertical.

The Von Mises stress through this bracket is shown in Fig. 23, where it can be seen that this bracket is fairly lightly loaded, with the maximum stress in the hooks around $350 \mathrm{MPa}$. The rest of the structure is even more lightly loaded, and therefore provides a good opportunity to minimize the weight using a cellular structure.

The results in Fig. 23 are used to generate the cellular topology, after which the cross-sectional area for each strut is optimized for minimum weight, subject to buckling and compatibility constraints. In this optimization, the radius of the struts is constrained to be between $0.3 \mathrm{~mm}$ and $1 \mathrm{~mm}$, the same constraints as used by Tang et al. (2015). ${ }^{6}$

The resulting cellular structure is shown in Fig. 24, which has a mass of $0.408 \mathrm{~kg}$-a $24 \%$ reduction compared to the cellular structure from Tang et al. (2015), which had a mass of $0.54 \mathrm{~kg}$. The difference is explained by the hooks and bolt holes being solid in that work, as well as by their use of a fine homogeneous cellular topology. It should also be noted that Tang et al. (2015) did not include buckling of the struts in their

\footnotetext{
6 Note that these constraints on the radius of the struts violate the minimum size requirements of the GE engine bracket, but are consistent with Tang et al. (2015).
}

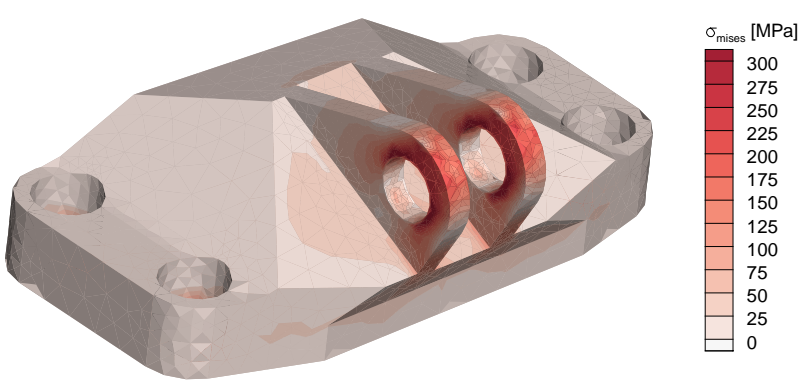

(a) The hooks are the highest stressed part of the bracket.

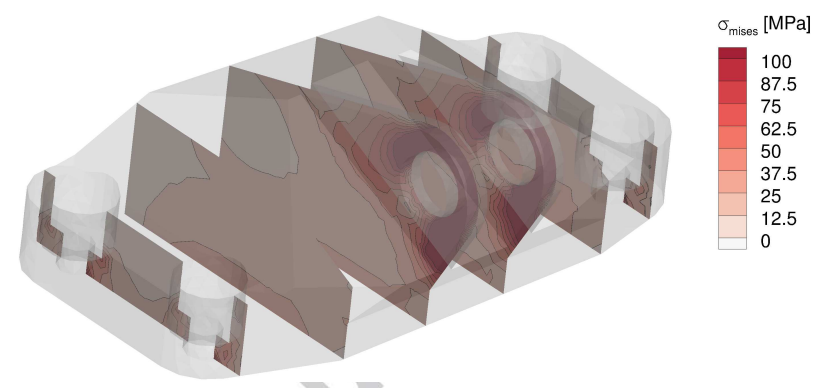

(b) The internals of the bracket experience minimal stress. Note that this figure's color legend has a different scale to figure (a).

Fig. 23: Von Mises stress through domain of GE bracket for load case in Fig. 22.

analysis, while that has a large influence on the masswithout including buckling, our analysis shows that the bracket would weigh $0.286 \mathrm{~kg}$. Use of stress-aligned cellular structures thus yields a large mass reduction. Note that the overall winner of the GE bracket challenge had a weight of $0.327 \mathrm{~kg}$ for a design that included four load cases. In that design, the outer topology of the bracket was allowed to change as well. In our design that topology stays fixed-it is expected that changes in outer topology can yield a further mass reduction in some cases. Allowing the outer topology to change as well in the optimization problem is a natural extension of this work, for instance using an approach similar to Ringertz (1985). Such a topology optimization approach could be used as the next step in a design phase to get higher performance for higher computational cost.

\section{Conclusion}

This paper presented an early-stage design methodology for cellular structures to be manufactured using additive manufacturing. In early-stage design phases it is important to characterize a part's performance and weight accurately with low computational cost, as potentially thousands of designs need to be evaluated on a system level. The design methodology therefore 

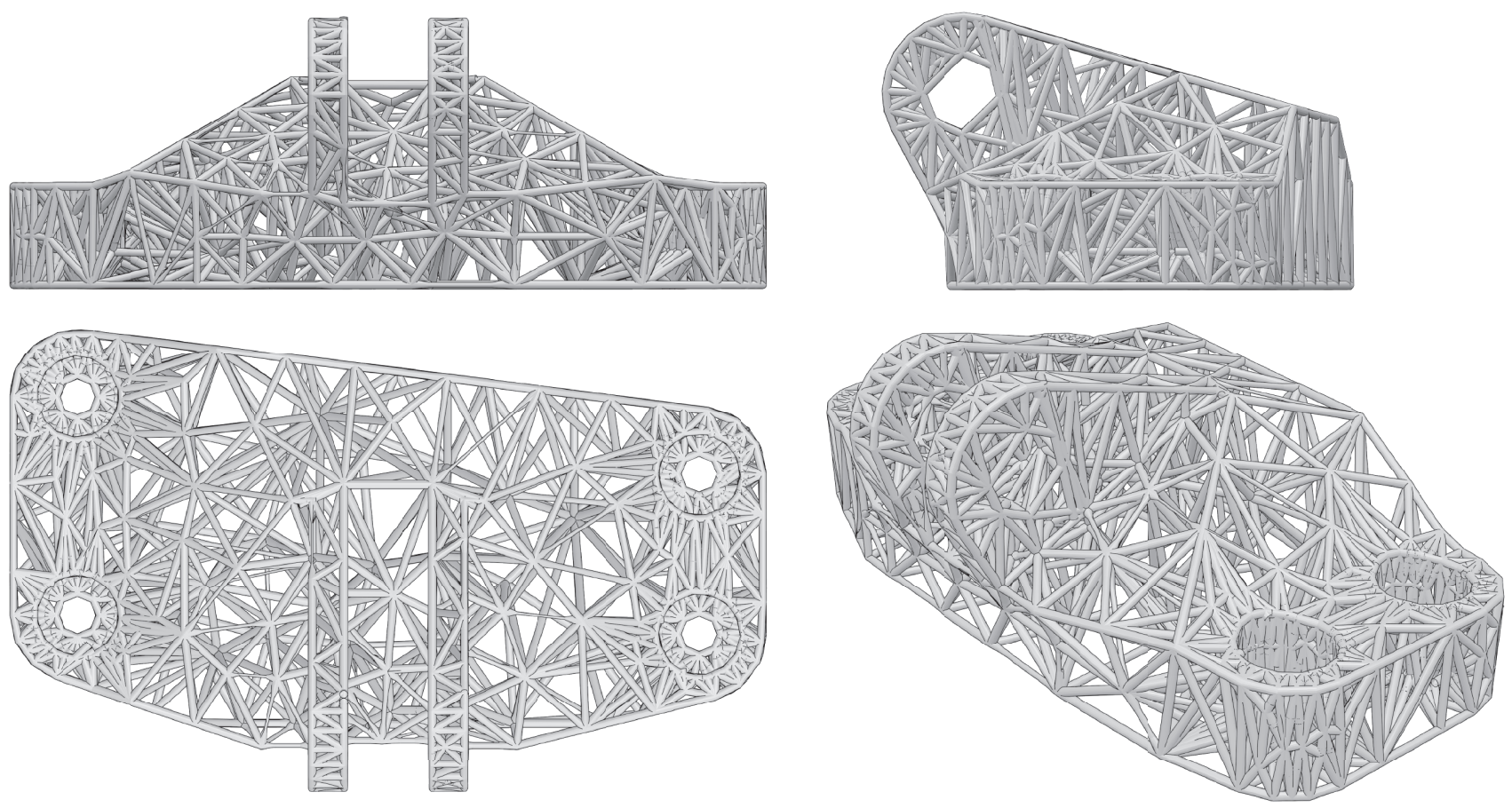

Fig. 24: Optimized cellular structure for aircraft bracket. This part weighs $0.408 \mathrm{~kg}$.

only uses one FEM solution, which is used to inform a Riemannian metric field. That metric field is employed to construct the cellular topology using adaptive meshing techniques, such that the cellular topology is fine near areas of high stress, coarse near areas of low stress, and is aligned with the stress direction. This approach also allows for including manufacturing constraints, such as minimum feature size and overhang angle constraints. Once the cellular topology is designed, the cross-sectional area of each strut is optimized for minimum weight subject to stress, compatibility, and buckling constraints.

This design methodology is applied to a small bracket to show the manufacturability of the part, where it was found that the design for manufacturability improves the manufacturability of the cellular structure substantially. Lastly, an aircraft bracket from the literature is redesigned to achieve a $24 \%$ weight improvement.

The approach demonstrated in this paper should be considered as the first step in design methodology for (large) additive manufactured structures. The next step (in the preliminary design phase) could be an approach where the outer topology of the part is allowed to change. Finally, in the detailed design phase, a levelset or density-based topology optimization approach can be used.

\section{Acknowledgements}

This work was supported in part by the NASA LEARN program grant number NNX14AC73A and by the SUTDMIT International Design Centre.

\section{Conflict of Interest}

On behalf of all authors, the corresponding author states that there is no conflict of interest.

\section{Replication of Results}

Parts of the results in this paper are reproducible using several packages which have been made available online. luteos. $\mathrm{jl}^{7}$ is used to solve the FEM problems in this paper, which uses the mesh data structure package divido.j1. ${ }^{8}$ Once the cellular structure is designed, the solid geometry is generated using intrico.j1. ${ }^{9}$

It is not possible to make the entire code-base available as it heavily relies on a metric-based mesherthroughout this paper feflo.a is used-which is not publicly available and still in development.

\footnotetext{
7 Available at github.com/mopg/luteos.jl.

8 Available at github.com/mopg/divido.jl.

9 Available at github.com/mopg/intrico.jl.
} 


\section{References}

Aage N, Andreassen E, Lazarov BS, Sigmund O (2017) Giga-voxel computational morphogenesis for structural design. Nature 550(7674):84, DOI 10.1038/ nature 23911

Bendsøe MP, Kikuchi N (1988) Generating optimal topologies in structural design using a homogenization method. Computer Methods in Applied Mechanics and Engineering 71(2):197-224, DOI 10.1016/ 0045-7825(88)90086-2

Bendsøe MP, Sigmund O (2013) Topology Optimization: Theory, Methods, and Applications. Springer Science \& Business Media

Clausen A, Wang F, Jensen JS, Sigmund O, Lewis JA (2015) Topology optimized architectures with programmable poisson's ratio over large deformations. Advanced Materials 27(37):5523-5527, DOI 10.1002/adma.201502485

Diaz MC, Hecht F, Mohammadi B, Pironneau O (1997) Anisotropic unstructured mesh adaptation for flows simulations. International Journal for Numerical Methods in Fluids 25:475-491, DOI 10.1007/ s00366-017-0513-2

Dorn WS, Gomory RE, Greenberg HJ (1964) Automatic design of optimal structures. Journal de Mecanique 3:25-52

Ejlebjerg Jensen K (2016) Anisotropic mesh adaptation and topology optimization in three dimensions. Journal of Mechanical Design 138(6):061401-1 - 0614018, DOI $10.1115 / 1.4032266$

Freund RM (2004) Truss design and convex optimization. Tech. rep., Massachusetts Institute of Technology

Gaynor AT, Guest JK (2016) Topology optimization considering overhang constraints: Eliminating sacrificial support material in additive manufacturing through design. Structural and Multidisciplinary Optimization 54(5):1157-1172, DOI 10.1007/ s00158-016-1551-x

Gibson L (1997) Cellular Solids: Structure and Properties. Cambridge University Press, Cambridge, England, United Kingdom

Graf GC, Chu J, Engelbrecht S, Rosen DW (2009) Synthesis methods for lightweight lattice structures. In: ASME 2009 International Design Engineering Technical Conferences and Computers and Information in Engineering Conference, American Society of Mechanical Engineers, pp 579-589, DOI 10.1115/ detc2009-86993

Groen JP, Sigmund O (2017) Homogenization-based topology optimization for high-resolution manufacturable microstructures. International Journal for
Numerical Methods in Engineering 113(8):11481163, DOI 10.1002/nme.5575

Guest JK (2008) Imposing maximum length scale in topology optimization. Structural and Multidisciplinary Optimization 37(5):463-473, DOI 10.1007/ s00158-008-0250-7

Guest JK, Prévost JH, Belytschko T (2004) Achieving minimum length scale in topology optimization using nodal design variables and projection functions. International Journal for Numerical Methods in Engineering 61(2):238-254, DOI 10.1002/nme.1064

Guo X, Zhang W, Zhong W (2014) Doing topology optimization explicitly and geometrically - a new moving morphable components based framework. Journal of Applied Mechanics 81(8):081009, DOI 10.1115/1. 4027609

Hamilton WR (1844) On a new species of imaginary quantities connected with a theory of quaternions. In: Proceedings of the Royal Irish Academy, vol 2, pp 424-434

Hecht F, Mohammadi B, Hecht F, Mohammadi B (1997) Mesh adaption by metric control for multiscale phenomena and turbulence. In: 35th Aerospace Sciences Meeting and Exhibit, Reno, Nevada, DOI 10.2514/6.1997-859

Hemp WS, Chan HSY (1966) Optimum design of pinjointed frameworks. Tech. Rep. 3632, Department of Engineering Science, University of Oxford

Kicher TP (1966) Optimum design - minimum weight versus fully stressed. Journal of the Structural Division 92(6):265-280

Kirsch U (1989) Optimal topologies of truss structures. Computer Methods in Applied Mechanics and Engineering 72(1):15-28, DOI 10.1016/0045-7825(89) 90119-9

Kirsch U (1993) Structural Optimization: Fundamentals and Applications. Springer Berlin Heidelberg, Heidelberg, Germany, DOI 10.1007/978-3-642-84845-2

Kolonay RM, Kobayashi MH (2015) Optimization of aircraft lifting surfaces using a cellular division method. Journal of Aircraft 52(6):2051-2063, DOI 10.2514/1.c033138

Langelaar M (2016) Topology optimization of 3d selfsupporting structures for additive manufacturing. Additive Manufacturing 12:60-70, DOI 10.1016/j. addma.2016.06.010

Loseille A (2014) Metric-orthogonal anisotropic mesh generation. Procedia Engineering 82:403-415, DOI 10.1016/j.proeng.2014.10.400

Loseille A, Alauzet F (2011a) Continuous mesh framework part I: Well-posed continuous interpolation error. SIAM Journal on Numerical Analysis 49(1):38 
60, DOI 10.1137/090754078

Loseille A, Alauzet F (2011b) Continuous mesh framework part II: Validations and applications. SIAM Journal on Numerical Analysis 49(1):61-86, DOI $10.1137 / 10078654 \mathrm{x}$

Loseille A, Lohner R (2010) Anisotropic adaptive simulations in aerodynamics. In: 48th AIAA Aerospace Sciences Meeting Including the New Horizons Forum and Aerospace Exposition, Orlando, Florida, DOI 10.2514/6.2010-169

Loseille A, Alauzet F, Menier V (2017) Unique cavitybased operator and hierarchical domain partitioning for fast parallel generation of anisotropic meshes. Computer-Aided Design 85:53-67, DOI 10.1016/j. cad.2016.09.008

Martins JRRA (2017) Chapter 19: Multidisciplinary design optimization of aerospace systems. In: Advances and Trends in Optimization with Engineering Applications, Society for Industrial and Applied Mathematics, pp 249-257, DOI 10.1137/1.9781611974683. $\operatorname{ch} 19$

Mazur M, Leary M, Sun S, Vcelka M, Shidid D, Brandt M (2016) Deformation and failure behaviour of ti$6 \mathrm{al}-4 \mathrm{v}$ lattice structures manufactured by selective laser melting $(\mathrm{slm})$. The International Journal of Advanced Manufacturing Technology 84(5-8):13911411, DOI 10.1007/s00170-015-7655-4

McMillan M, Jurg M, Leary M, Brandt M (2015) Programmatic lattice generation for additive manufacture. Procedia Technology 20:178-184, DOI 10.1016/ j.protcy.2015.07.029

Michell AGM (1904) The limits of economy of material in frame structure. Philosophical Magazine 8(6):589597, DOI 10.1080/14786440409463229

Norato J, Bell B, Tortorelli D (2015) A geometry projection method for continuum-based topology optimization with discrete elements. Computer Methods in Applied Mechanics and Engineering 293:306-327, DOI 10.1016/j.cma.2015.05.005

Ohsaki M (2011) Optimization of Finite Dimensional Structures. CRC Press/Taylor \& Francis, Boca Raton

Opgenoord MMJ (2018) Transonic flutter prediction and aeroelastic tailoring for next-generation transport aircraft. PhD thesis, Department of Aeronautics \& Astronautics, Massachusetts Institute of Technology

Osher SJ, Santosa F (2001) Level set methods for optimization problems involving geometry and constraints: I. frequencies of a two-density inhomogeneous drum. Journal of Computational Physics 171(1):272-288, DOI 10.1006/jcph.2001.6789
Pantz O, Trabelsi K (2008) A post-treatment of the homogenization method for shape optimization. SIAM Journal on Control and Optimization 47(3):1380 1398, DOI 10.1137/070688900

Poulsen TA (2003) A new scheme for imposing a minimum length scale in topology optimization. International Journal for Numerical Methods in Engineering 57(6):741-760, DOI 10.1002/nme.694

Qian X (2017) Undercut and overhang angle control in topology optimization: A density gradient based integral approach. International Journal for Numerical Methods in Engineering 111(3):247-272, DOI $10.1002 / \mathrm{nme} .5461$

Redwood B, Schöffer F, Garret B (2017) The 3D Printing Handbook: Technologies, Design and Applications. 3D Hubs, Amsterdam, The Netherlands

Rehme O (2010) Cellular design for laser freeform fabrication. PhD thesis, Technical University Hamburg, Göttingen

Ringertz UT (1985) On topology optimization of trusses. Engineering Optimization 9(3):209-218, DOI 10.1080/03052158508902514

Rozvany GIN (2001) On design-dependent constraints and singular topologies. Structural and Multidisciplinary Optimization 21(2):164-172, DOI 10.1007/ s001580050182

Schmidtke K, Palm F, Hawkins A, Emmelmann C (2011) Process and mechanical properties: Applicability of a scandium modified al-alloy for laser additive manufacturing. Physics Procedia 12:369-374, DOI 10.1016/j.phpro.2011.03.047

Sethian JA, Wiegmann A (2000) Structural boundary design via level-set and immersed interface methods. Journal of Computational Physics 163(2):489-528, DOI 10.1006/jcph.2000.6581

Shames I, Pitarresi JM (2000) Introduction to Solid Mechanics. Prentice Hall, Upper Saddle River, NJ

Shidid D, Leary M, Choong P, Brandt M (2016) Justin-time design and additive manufacture of patientspecific medical implants. Physics Procedia 83:4-14, DOI 10.1016/j.phpro.2016.08.002

Sigmund O, Aage N, Andreassen E (2016) On the (Non-)optimality of michell structures. Structural and Multidisciplinary Optimization 54(2):361-373, DOI 10.1007/s00158-016-1420-7

Soon SC, Cockburn B, Stolarski HK (2009) A hybridizable discontinuous galerkin method for linear elasticity. International Journal for Numerical Methods in Engineering 80(8):1058-1092, DOI 10.1002/nme. 2646

Stanford BK, Dunning PD (2015) Optimal topology of aircraft rib and spar structures under aeroelastic loads. Journal of Aircraft 52(4):1298-1311, DOI 
10.2514/1.c032913

Tang Y, Kurtz A, Zhao YF (2015) Bidirectional evolutionary structural optimization (beso) based design method for lattice structure to be fabricated by additive manufacturing. Computer-Aided Design 69:91101, DOI 10.1016/j.cad.2015.06.001

Townsend S, Picelli R, Stanford B, Kim HA (2018) Structural optimization of platelike aircraft wings under flutter and divergence constraints. AIAA Journal 56(8):3307-3319, DOI 10.2514/1.j056748

Van Dijk NP, Maute K, Langelaar M, van Keulen F (2013) Level-set methods for structural topology optimization: A review. Structural and Multidisciplinary Optimization 48(3):437-472, DOI 10.1007/ s00158-013-0912-y

Vanderplaats GN, Moses F (1972) Automated design of trusses for optimum geometry. Journal of the Structural Division 98(3):671-690

Vaneker THJ (2017) The role of design for additive manufacturing in the successful economical introduction of am. Procedia CIRP 60:181-186, DOI 10.1016/j.procir.2017.02.012

Wächter A, Biegler LT (2006) On the implementation of an interior-point filter line-search algorithm for large-scale nonlinear programming. Mathematical Programming 106(1):25-57, DOI 10.1007/ s10107-004-0559-y

Watts S, Tortorelli DA (2018) A geometric projection method for designing three-dimensional open lattices with inverse homogenization. International Journal for Numerical Methods in Engineering 113(8):14111411, DOI 10.1002/nme.5761

Wie B (2008) Space Vehicle Dynamics and Control. American Institute of Aeronautics and Astronautics (AIAA), Reston, VA, DOI 10.2514/4.860119

Yano M, Darmofal DL (2012) An optimization-based framework for anisotropic simplex mesh adaptation. Journal of Computational Physics 231(22):76267649, DOI 10.1016/j.jcp.2012.06.040

Zhou K (2012) Topology optimization of truss-like continuum structures for natural frequencies. Structural and Multidisciplinary Optimization 47(4):613-619, DOI 10.1007/s00158-012-0870-9 